\title{
The pinching method for Galactic cosmic ray positrons: Implications in the light of precision measurements
}

\author{
M. Boudaud ${ }^{1,4, \star}$, E. F. Bueno ${ }^{3}$, S. Caroff ${ }^{2}$, Y. Genolini ${ }^{1}$, V. Poulin ${ }^{1,5}$, \\ V. Poireau ${ }^{2}$, A. Putze ${ }^{1,2}$, S. Rosier ${ }^{2}$, P. Salati $^{1}$, and M. Vecchi ${ }^{3}$ \\ ${ }^{1}$ Laboratoire d'Annecy-le-Vieux de Physique théorique (LAPTh), CNRS and Université Savoie Mont Blanc, 9 Chemin de Bellevue, \\ BP 110, 74941 Annecy-le-Vieux, France \\ e-mail: [mathieu.boudaud; yoann.genolini; vivian.poulin]@lapth.cnrs.fr \\ 2 Laboratoire d'Annecy-le-Vieux de Physique des Particules (LAPP), CNRS/IN2P3 and Université Savoie Mont Blanc, \\ 9 Chemin de Bellevue, BP 110, 74941 Annecy-le-Vieux, France \\ e-mail: sami.caroff@lapp.in2p3.fr \\ 3 Instituto de Física de São Carlos (IFSC), Universidade de São Paulo, CP 369, 13560-970, São Carlos, SP, Brazil \\ ${ }^{4}$ Laboratoire de Physique Théorique et Hautes Énergies (LPTHE), CNRS and Université Pierre et Marie Curie, Boîte 126, \\ T13-14 4ème étage, 4 place Jussieu, 75252 Paris Cedex 05, France \\ 5 Institute for Theoretical Particle Physics and Cosmology (TTK), RWTH Aachen University, 52056 Aachen, Germany
}

Received 22 December 2016 / Accepted 4 May 2017

\begin{abstract}
Context. Two years ago, the AMs-02 collaboration released the most precise measurement of the cosmic ray positron flux. In the conventional approach, in which positrons are considered as purely secondary particles, the theoretical predictions fall way below the data above $10 \mathrm{GeV}$. One suggested explanation for this anomaly is the annihilation of dark matter particles, the so-called weakly interactive massive particles (WIMPs), into standard model particles. Most analyses have focused on the high-energy part of the positron spectrum, where the anomaly lies, disregarding the complicated $\mathrm{GeV}$ low-energy region where Galactic cosmic ray transport is more difficult to model and solar modulation comes into play.

Aims. Given the high quality of the latest measurements by AMS-02, it is now possible to systematically re-examine the positron anomaly over the entire energy range, this time taking into account transport processes so far neglected, such as Galactic convection or diffusive re-acceleration. These might impact somewhat on the high-energy positron flux so that a complete and systematic estimate of the secondary component must be performed and compared to the AMS-02 measurements. The flux yielded by WIMPs also needs to be re-calculated more accurately to explore how dark matter might source the positron excess.

Methods. We devise a new semi-analytical method to take into account transport processes thus far neglected, but important below a few GeV. It is essentially based on the pinching of inverse Compton and synchrotron energy losses from the magnetic halo, where they take place, inside the Galactic disc. The corresponding energy loss rate is artificially enhanced by the so-called pinching factor, which needs to be calculated at each energy. We have checked that this approach reproduces the results of the Green function method at the per mille level. This new tool is fast and allows one to carry out extensive scans over the cosmic ray propagation parameters. Results. We derive the positron flux from sub-GeV to $\mathrm{TeV}$ energies for both gas spallation and dark matter annihilation. We carry out a scan over the cosmic ray propagation parameters, which we strongly constrain by requiring that the secondary component does not overshoot the AMS-02 measurements. We find that only models with large diffusion coefficients are selected by this test. We then add to the secondary component the positron flux yielded by dark matter annihilation. We carry out a scan over WIMP mass to fit the annihilation cross-section and branching ratios, successively exploring the cases of a typical beyond-the-standard-model WIMP and an annihilation through light mediators. In the former case, the best fit yields a $p$-value of $0.4 \%$ for a WIMP mass of $264 \mathrm{GeV}$, a value that does not allow to reproduce the highest energy data points. If we require the mass to be larger than $500 \mathrm{GeV}$, the best-fit $\chi^{2}$ per degree of freedom always exceeds a value of 3 . The case of light mediators is even worse, with a best-fit $\chi^{2}$ per degree of freedom always larger than 15.

Conclusions. We explicitly show that the cosmic ray positron flux is a powerful and independent probe of Galactic cosmic ray propagation. It should be used as a complementary observable to other tracers such as the boron-to-carbon ratio. This analysis shows also that the pure dark matter interpretation of the positron excess is strongly disfavoured. This conclusion is based solely on the positron data, and no other observation, such as the antiproton flux or the CMB anisotropies, needs to be invoked.
\end{abstract}

Key words. astroparticle physics - cosmic rays - dark matter - elementary particles

\section{Introduction}

The cosmic ray (CR) positron flux has been measured with unprecedented accuracy by the AMs- 02 collaboration (Aguilar et al. 2014). This observation is of paramount importance in several respects. To start with, it provides an insight into

\footnotetext{
$\star$ All authors are members of the Cosmic Ray Alpine Collaboration.
}

the mechanisms that create positrons inside the Milky Way. For a long time, CR positrons have been thought to be exclusively secondary species originating from the spallation of the gas lying in the Galactic disc. The first experimental hints for a deviation from the conventional scenario came from the data collected by the High-Energy Antimatter Telescope (HEAT) collaboration (Barwick et al. 1997; DuVernois et al. 2001; Beatty et al. 2004), 
but the existence of a positron anomaly has been firmly established by Adriani et al. (2009) who reported an excess in the positron fraction measured up to $100 \mathrm{GeV}$ by the Payload for Antimatter Matter Exploration and Light-nuclei Astrophysics (PAMELA) satellite. Recently, the Alpha Magnetic Spectrometer (AMS-02) has initiated a new era of precision measurements with the release of high-quality data, in particular on the positron fraction (Aguilar et al. 2013; Accardo et al. 2014) and positron flux (Aguilar et al. 2014) up to $500 \mathrm{GeV}$. The AMs-02 results definitely confirm that, in addition to the secondary component, a new ingredient is at play in the cosmic positron radiation.

These measurements have an obvious connection with the long standing problem of the astronomical dark matter (DM). The nature of this enigmatic component, which contributes a fraction of $\sim 27 \%$ to the energy budget of the Universe, is still unresolved. The most commonly accepted hypothesis is based on a weakly interacting massive particle, dubbed WIMP, whose existence is predicted in most extensions of the high-energy physics standard model. A distinctive feature of these species is that they are produced in the early Universe, through self-annihilation, with a relic abundance in close agreement with the cosmological observations. In this approach, weakly interactive massive particles (WIMPs) pervade the Galactic halo wherein they still pair annihilate today, yielding positrons among other standard model particles. The positron anomaly has triggered a feverish activity insofar as it could be interpreted as the imprint left by DM species on the CR positron spectrum. Many investigations explored whether or not WIMPs might be the source of that anomaly. We refer the reader to the analysis by (Di Mauro et al. 2014, 2016; Lin et al. 2015; Boudaud et al. 2015) and references therein. The vast majority of these studies are focused on the high-energy part of the positron spectrum, above $10 \mathrm{GeV}$. Below this energy, solar modulation comes into play and complicates the interpretation of the data. Moreover, Galactic convection, diffusive re-acceleration, and positron annihilation on interstellar gas must be taken into account in addition to space diffusion. Finally, energy losses, which play a key role in the propagation of positrons, are mostly concentrated inside the Galactic disc whereas they extend all over the magnetic halo at high energy.

Besides the complexity of modelling CR transport below a few $\mathrm{GeV}$, there is also the implicit but widespread assumption that DM should essentially show up at high energies. The corollary of that standpoint is that some yet-to-be-determined astrophysical sources should be operating at low energies so as to produce the positron flux at the observed level. Pulsars (Hooper et al. 2009; Profumo 2012; Linden \& Profumo 2013) or spallations inside the supernova-driven shock waves (Blasi 2009; Mertsch \& Sarkar 2014) are two examples of such possibilities. But if additional processes need to be invoked to explain the low-energy part of the positron data, nothing precludes them from coming into play also at higher energies. There is nothing special taking place at a scale of a few $\mathrm{GeV}$, and processes known to be active at low energy are expected to contribute all over the positron spectrum. Of course, looking for a DM solution of the positron anomaly by fitting the WIMP cross-section and mass to the high-energy part of the data is tantalising. This has actually been the subject of numerous studies since the discovery of the positron excess by PAMELA. But these analyses are based on the prejudice that low-energy positron data are not relevant to DM, an unwarranted assumption that might introduce biases. For instance, should some WIMP parameters provide a good fit, one might be left with the illusion that the positron excess is a smoking gun signature of the presence of DM species inside the Galaxy. One should instead conclude that, even though the data actually support the WIMP hypothesis, they cannot be considered as compelling evidence for particle DM. For this, DM annihilation and gas spallation by CR nuclei must be proved to be the only sources of high-energy positrons while, at the same time, other production mechanisms are assumed to operate at low energy. Another flaw in this approach is the risk that a WIMP model that fits well the positron excess above a few $\mathrm{GeV}$ could simultaneously be a poor match to the low-energy data, overshooting them for instance. Establishing the DM hypothesis requires then to derive the positron flux over the entire accessible energy range, and not just on its high-energy part.

These considerations have led us to reinvestigate the problem of the positron anomaly over the entire energy range covered by the AMS-02 data. In order to test the DM hypothesis, we have computed the interstellar positron flux yielded at the Earth by (i) the spallation of interstellar gas by $\mathrm{CR}$ protons and helium nuclei and (ii) WIMP annihilation. To do so, we have conceived a new semi-analytical method to cope with transport processes thus far neglected but important below a few GeV. This new tool also allows us to carry out extensive scans over the CR propagation parameters. The main point of novelty consists in the analytic treatment of the energy losses suffered by cosmic ray positrons in the magnetic halo; the corresponding effect being artificially enhanced by the so-called pinching factor, that shifts the energy losses from the magnetic halo, where they actually take place, inside the Galactic disc. An essential benefit is a faster runtime in comparison to a fully numerical approach.

We have also improved the calculation of the background of secondary positrons by using the new measurements of the $\mathrm{CR}$ proton and helium fluxes by the AMS- 02 collaboration (Aguilar et al. 2015b,a). There is a hardening above $\sim 300 \mathrm{GeV}$ that leads to a slight increase of the positron yield from gas spallation. We have overcome the difficulty arising from solar modulation by using the value of the Fisk potential inferred by Ghelfi et al. (2016) from their analysis of the variations of the top-of-the-atmosphere (TOA) proton flux over the recent past.

Equipped with the pinching method, we have improved upon the analysis by Lavalle et al. (2014) by carrying out a scan over the CR propagation models found by Maurin et al. (2001) to be compatible with the $\mathrm{B} / \mathrm{C}$ ratio, and by deriving for each of them the positron flux yielded by gas spallation. We have finally investigated the DM solution to the positron anomaly by calculating, for each of the surviving CR models, the yield from an annihilating WIMP to which we have added the secondary positron background. The positron flux is derived over the same energy range as for the AMS-02 data. We have performed a scan over WIMP mass and explored the possibility of mixed annihilation channels. At fixed WIMP mass, we have derived the best-fit values of the annihilation cross-section and branching ratios. We have considered DM particles annihilating either directly in standard model particles or through light mediators.

The article is organised according the points sketched above. The pinching method, which is paramount to this investigation, is detailed in Sect. 2. We apply this new tool in Sect. 3 to investigate the implications on the positron flux of CR transport processes so far neglected at high energies. The astrophysical background of secondary positrons is discussed in Sect. 3.1 while the positron flux yielded by DM species is presented in Sect. 3.2. We then constrain in Sect. 4 the CR propagation parameters, requiring that they do not lead to a flux of secondary positrons in excess of the measurements. The scan procedure is exposed and results in a sharp selection of the CR models. The DM interpretation of the AMS-02 data is presented in Sect. 5. In Sect. 6 
we investigate the robustness of our results and explore a few sources of uncertainties. We finally conclude in Sect. 7.

\section{Propagation of Galactic cosmic ray positrons with the pinching method}

In this section, we recall the basics of the propagation of CRs in the Galaxy. We first present the transport equation and its semianalytical resolution. We then introduce a new method, referred to hereafter as the pinching method, to semi-analytically solve the transport equation for electrons and positrons when all propagation effects are simultaneously taken into account.

\subsection{The transport equation of $C R s$}

During their journey across the Galaxy, CRs are affected by many processes as a result of their interactions with the Galactic magnetic field (GMF) and the interstellar medium (ISM). Despite the strength of the magnetic turbulence, Fick's law still holds (Casse et al. 2002). Hence, the scattering of CRs on the GMF can be described by a random walk and modelled by a diffusion process in space. In this work we choose a homogeneous and isotropic diffusion coefficient $K(E)=\beta K_{0}(\mathcal{R} / 1 \mathrm{GV})$ where $\beta$ is the velocity of the particle and $\mathcal{R}$ the rigidity related to its momentum $p$ and its charge $q$ by $\mathcal{R}=p / q$. On top of this, the diffusion centers move with the Alfvèn waves velocity $V_{\mathrm{a}}$. Thus, CRs undergo a diffusive reacceleration (DR) thanks to the second-order Fermi mechanism. This process can be modelled by a diffusion in energy space with coefficient $D(E)=(2 / 9) V_{\mathrm{a}}^{2} E^{2} \beta^{4} / K(E)$. Moreover, CRs can interact with the ISM, leading to: i) energy losses from Coulomb interaction and ionisation, with respective rates $b_{\text {coul }}$ and $b_{\text {ioni }}$; and ii) their destruction at a rate $\Gamma$. In addition, electrons and positrons (loosely dubbed electrons hereafter except when explicitly mentioned) lose energy by bremsstrahlung, synchrotron emission, and inverse Compton (IC) scattering when they interact with the interstellar radiation field (ISRF), at respective rates $b_{\text {brem }}, b_{\text {sync }}$, and $b_{\text {IC }}$. Following the procedure described in Delahaye et al. (2010), we consider IC scattering in the relativistic regime and make use of the mean value of the GMF $\langle B\rangle=1 \mu \mathrm{G}$ (Ferrière 2001). Finally, CRs are blown by the Galactic wind (GW) produced by supernova remnant explosions in the Galactic disc. We assume the GW to be homogeneous and perpendicular to the Galactic disc, with velocity $\boldsymbol{V}_{\mathrm{c}}=\operatorname{sign}(z) V_{\mathrm{c}} \boldsymbol{e}_{z}$. This process leads to the adiabatic cooling of CRs, which enters as an additional term in the energy loss rate $b_{\text {adia }}$. The total energy loss rate $b(E) \equiv \mathrm{d} E / \mathrm{d} t$ is then simply the sum of all the loss processes (their explicit expression can be found in Strong \& Moskalenko 1998 and Moskalenko \& Strong 1998).

Following the work of Maurin et al. (2001, and references therein), we assume the Galaxy to be axisymmetric and describe it by the two-zone model. The first zone, within which ISM is homogeneously distributed, represents the Galactic disc of half-height $h=100 \mathrm{pc}$. Atomic densities are taken to be $n_{\mathrm{H}}=0.9 \mathrm{~cm}^{-3}$ and $n_{\mathrm{He}}=0.1 \mathrm{~cm}^{-3}$. It is embedded inside a much larger second zone, namely the magnetic halo $(\mathrm{MH})$, of half-height $L$ lying between 1 and $15 \mathrm{kpc}$. Both zones share the radius $R=20 \mathrm{kpc}$. In practice, we assume the space diffusion, as well as the energy losses from synchroton emission and IC scattering, to lie in the whole magnetic halo. On the other hand, DR, bremsstrahlung, Coulomb interaction, ionisation, and destruction take place only in the Galactic disc where the matter of the ISM is concentrated (Ptuskin et al. 1997).
Hence, we split the energy losses $b(E, z)$ into a disc component $b_{\text {disc }} \equiv b_{\text {coul }}+b_{\text {brem }}+b_{\text {ioni }}+b_{\text {adia }}$ that includes the mechanisms that take place only in the Galactic disc, and a halo component $b_{\text {halo }} \equiv b_{\text {IC }}+b_{\text {sync }}$ considering those that take place in the whole magnetic halo (including the disc). We impose a vanishing density of CRs outside the MH of the Galaxy.

Under a steady state and thin disc approximation, the density of CRs per unit of space and energy $\psi \equiv \mathrm{d} N / \mathrm{d}^{3} x \mathrm{~d} E$ obeys the transport equation

$$
\begin{aligned}
\boldsymbol{\nabla} & \cdot\left[\boldsymbol{V}_{\mathrm{c}} \psi(E, r, z)-K(E) \boldsymbol{\nabla} \psi(E, r, z)\right] \\
& +\partial_{E}\left[b(E, z) \psi(E, r, z)-2 h \delta(z) D(E) \partial_{E} \psi(E, r, z)\right] \\
& +2 h \delta(z) \Gamma \psi=Q(E, r, z),
\end{aligned}
$$

where $Q$ represents the injection rate of CRs in the Galaxy.

$\mathrm{CR}$ nuclei lose energy only in the Galactic disc (i.e. $b_{\text {halo }}^{\text {nuc }}=$ $0)$. In this case, the transport Eq. (1) can be solved via the semianalytical scheme introduced in Maurin et al. (2001). More precisely, the CR density $\psi$ is expanded on the basis of the firstorder Bessel functions $J_{0}$ such that

$$
\psi(E, r, z)=\sum_{i=1}^{\infty} J_{0}\left(\alpha_{i} \frac{r}{R}\right) P_{i}(E, z),
$$

where $\alpha_{i}$ are the zero of the Bessel function $J_{0}$. The transport Eq. (1) consequently becomes

$$
\begin{aligned}
& \partial_{z}\left[V_{\mathrm{c}}(z) P_{i}\right]-K(E) \partial_{z}^{2} P_{i}+K(E)\left(\frac{\alpha_{i}}{R}\right)^{2} P_{i} \\
& \quad+2 h \delta(z) \partial_{E}\left[b(E) P_{i}-D(E) \partial_{E} P_{i}\right]+2 h \delta(z) \Gamma P_{i}=Q_{i}(E, z),(3)
\end{aligned}
$$

where $Q_{i}(E, z)$ are the Bessel transform coefficients of the source term $Q(E, r, z)$. Equation (3) is then reduced to a second-order ordinary differential equation for the function $P_{i}(E, z=0)$ with respect to the energy $E$, and can be solved numerically using a Cranck-Nicholson algorithm. Finally, the CR flux at the Earth is given by $\Phi(E, \odot)=v / 4 \pi \psi(E, \odot)$ where $r_{\odot}=8.5 \mathrm{kpc}$. For more details on the resolution method, we refer the reader to Maurin et al. (2001). In this way, previous authors used the semianalytical method to determine 1623 sets of propagation parameters constrained by the boron over carbon ratio $\mathrm{B} / \mathrm{C}$ measurements. This enabled them to derive in Donato et al. (2004) the benchmark MIN, MED, and MAX propagation models presented in Table 1.

In the case of electrons, the semi-analytical resolution of the transport equation, as it is, is not possible. Indeed, the difficulty comes from the fact that electrons lose energy in the Galactic disc as well as in the whole magnetic halo. In the thin disc approximation, the energy loss rate can be written $b(E, z)=2 h \delta(z) b_{\text {disc }}(E)+b_{\text {halo }}(E)$, but the presence of the term $b_{\text {halo }}$ prevents direct semi-analytical resolution of Eq. (3). Therefore, numerical codes have been adopted to predict the flux of electrons at the Earth. An alternative way, often used in the literature, is to focus only on high-energy electrons $(E>$ few $\mathrm{GeV})$. In this case, as shown in Delahaye et al. (2009), the dominant propagation processes are the space diffusion and the halo energy losses $\left(b_{\text {sync }}\right.$ and $\left.b_{\text {IC }}\right)$. The high-energy approximation consists thus in neglecting the DR, the convection, the disc energy losses $b_{\text {disc }}$, and the destruction of CRs. Hence, the high-energy transport equation can be written

$-K(E) \Delta \psi+\partial_{E}[b(E) \psi]=Q(E, r, z)$,

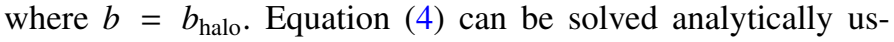
ing the pseudo-time method introduced by Baltz \& Edsjö (1999) 
Table 1. Benchmark MIN, MED, and MAX sets of propagation parameters introduced in Donato et al. (2004).

\begin{tabular}{cccccc}
\hline \hline Case & $\delta$ & $K_{0}\left[\mathrm{kpc}^{2} / \mathrm{Myr}\right]$ & $L[\mathrm{kpc}]$ & $V_{\mathrm{c}}\left[\mathrm{km} \mathrm{s}^{-1}\right]$ & $V_{\mathrm{a}}\left[\mathrm{km} \mathrm{s}^{-1}\right]$ \\
\hline MIN & 0.85 & 0.0016 & 1 & 13.5 & 22.4 \\
MED & 0.70 & 0.0112 & 4 & 12 & 52.9 \\
MAX & 0.46 & 0.0765 & 15 & 5 & 117.6 \\
\hline
\end{tabular}

and its solution can be expressed in terms of Bessel functions (Delahaye et al. 2008), where the Bessel coefficients evaluated at $z=0$ are given by

$P_{i}(E, 0)=\frac{-1}{b(E)} \int_{E}^{+\infty} \mathrm{d} E_{\mathrm{S}} B_{i}\left(E, E_{\mathrm{S}}\right)$,

where

$B_{i}\left(E, E_{\mathrm{S}}\right)=\sum_{n=2 m+1}^{+\infty} Q_{i, n}\left(E_{\mathrm{S}}\right) \exp \left[-C_{i, n} \lambda_{\mathrm{D}}^{2}\right]$.

The function $Q_{i, n}$ is the Fourrier transform of $Q_{i}(E, z)$ defined as

$Q_{i, n}(E)=\frac{1}{L} \int_{-L}^{L} \mathrm{~d} z \varphi_{n}(z) Q_{i}(E, z)$,

where $\varphi_{n}(z)=\cos \left(n k_{0} z\right)$ with $k_{0}=\pi / 2 L$. The coefficient $C_{i, n}$ is defined as

$C_{i, n}=\frac{1}{4}\left[\left(\frac{\alpha_{i}}{R}\right)^{2}+\left(n k_{0}\right)^{2}\right]$.

Finally, the diffusion length $\lambda_{\mathrm{D}}$ is related to the space diffusion coefficient $K$ and the energy loss rate $b$ by the expression

$\lambda_{\mathrm{D}}^{2}\left(E, E_{\mathrm{S}}\right)=4 \int_{E_{\mathrm{S}}}^{E} \mathrm{~d} E^{\prime} \frac{K\left(E^{\prime}\right)}{b\left(E^{\prime}\right)}$.

We note that the density $\psi$ at the Earth can be written as

$\psi(E, \odot)=\frac{-1}{b(E)} \int_{E}^{+\infty} \mathrm{d} E_{\mathrm{S}} \mathcal{I}\left(\lambda_{D}\right) Q\left(E_{\mathrm{S}}, \odot\right)$,

where the halo integral $\mathcal{I}$ is defined as

$\mathcal{I}\left(\lambda_{\mathrm{D}}\right)=\sum_{i=1}^{+\infty} J_{0}\left(\alpha_{i} \frac{r}{R}\right) \frac{B_{i}\left(E, E_{\mathrm{S}}\right)}{Q\left(E_{\mathrm{S}}, \odot\right)}$.

The flux at the Earth can then be computed for secondary electrons from proton and helium spallation (Delahaye et al. 2009; Boudaud et al. 2015), as well as for primary electrons from the ones produced by DM particle annihilations (Delahaye et al. 2008; Boudaud et al. 2015) and astrophysical objects like pulsars (Boudaud et al. 2015). One can then perform comparisons with data, which have led to the discovery of a high-energy positron excess requiring the presence of a dominant primary component above approximately $10 \mathrm{GeV}$. The high-energy approximation is often used in the literature to derive conclusions for energies above that value. However, it is not obvious that the low-energy propagation effects (DR, convection, and energy losses in the Galactic disc) can be safely neglected, especially in the era of the AMS-02 high-accuracy measurements. Furthermore, due to high statistics, the region below $10 \mathrm{GeV}$ is affected by the lowest experimental uncertainties and could thus provide the strongest constraints. These considerations led us to develop a new theoretical solution for the propagation of electrons over the energy range covered by AMS- 02 . This method dubbed the pinching method is described in the following section.

\subsection{The pinching method}

At first sight, it seems that the semi-analytical method cannot be used to solve Eq. (1) when energy losses take place simultaneously in the $\mathrm{MH}$ and in the Galactic disc. The trick to overcome this issue is to force the halo energy losses to take place, in an effective way, only in the Galactic disc. In other words, it consists in replacing the term $b_{\text {halo }}$ in the transport Eq. (1) with an effective term $2 h \delta(z) b_{\text {halo }}^{\text {eff }}$ while keeping the same solution $\psi$. By doing so, it will be possible to rewrite Eq. (1) in the form of Eq. (3) and to apply the Crank-Nicholson algorithm to solve it. This procedure consists thus in pinching the halo energy losses inside the disc, hence the name pinching method.

The function $b_{\text {halo }}^{\text {eff }}$ depends on all the propagation effects that electrons undergo. Nevertheless, from a few $\mathrm{GeV}$ to $1 \mathrm{TeV}$, halo energy losses and space diffusion are the dominant propagation processes (Delahaye et al. 2009). Hence, at first order, we can reasonably neglect other processes and determine $b_{\text {halo }}^{\text {eff }}$ using the high-energy approximation, that is, Eq. (4). This approximation may not be completely valid for energies below a few $\mathrm{GeV}$ where other effects come into play and are expected to affect the calculation of $b_{\text {halo }}^{\text {eff }}$. But the more dominant these processes are, the less important halo energy losses turn out to be, meaning that the precise value of the pinching factor does not matter at low energies.

Let us start with the pedagogical case of a monochromatic source of electrons $Q(E, r, z)=\delta\left(E-E_{\mathrm{S}}\right) Q(r, z)$. In order to determine $b_{\text {halo }}^{\text {eff }}$, we compute first the exact high-energy solution $\psi^{h}$ using the pseudo-time method described above. The index $h$ means that $\psi^{h}$ is solution of Eq. (4) where IC and synchrotron energy losses are distributed in the whole $\mathrm{MH}$. In that case, the electron density $\psi^{h}$ at $z=0$ is given by

$\psi^{h}(E, r, 0)=\sum_{i=1}^{+\infty} J_{0}\left(\alpha_{i} \frac{r}{R}\right) P_{i}^{h}(E, 0)$

where $P_{i}^{h}(E, 0)$ is given by the expression (5).

In a second step, we introduce $\psi^{d}$, the solution of the highenergy equation

$-K(E) \Delta \psi^{d}+2 h \delta(z) \partial_{E}\left[b_{\text {halo }}^{\mathrm{eff}} \psi^{d}\right]=Q(E, r, z)$,

where IC and synchrotron energy losses are confined to the disc. The condition $\psi^{h}(E, r, 0)=\psi^{d}(E, r, 0)$ enables us then to determine the function $b_{\text {halo }}^{\text {eff }}$ such that

$b_{\text {halo }}^{\text {eff }}\left(E, E_{\mathrm{S}}, r\right)=\xi\left(E, E_{\mathrm{S}}, r\right) b_{\text {halo }}(E)$,

where we introduced the pinching factor $\xi\left(E, E_{\mathrm{S}}, r\right)$, given by the expression

$\xi\left(E, E_{\mathrm{S}}, r\right)=\frac{1}{\psi^{h}(E, r, 0)} \sum_{i=1}^{+\infty} J_{0}\left(\alpha_{i} \frac{r}{R}\right) \xi_{i}\left(E, E_{\mathrm{S}}\right) P_{i}(E, 0)$, 
with

$\xi_{i}\left(E, E_{\mathrm{S}}\right)=\frac{1}{B_{i}\left(E, E_{\mathrm{S}}\right)}\left[J_{i}\left(E_{\mathrm{S}}\right)+4 k_{i}^{2} \int_{E}^{E_{\mathrm{S}}} \mathrm{d} E^{\prime} \frac{K\left(E^{\prime}\right)}{b_{\text {halo }}\left(E^{\prime}\right)} B_{i}\left(E^{\prime}, E_{\mathrm{S}}\right)\right]$.

The coefficient $J_{i}$ and $k_{i}$ are given by

$k_{i}^{2}=\frac{S_{i}}{8 h} \operatorname{coth}\left(\frac{S_{i} L}{2}\right)$

and

$J_{i}\left(E_{\mathrm{S}}\right)=\frac{1}{h} \int_{0}^{L} \mathrm{~d} z_{\mathrm{S}} \mathcal{F}_{i}\left(z_{\mathrm{S}}\right) Q_{i}\left(E_{\mathrm{S}}, z_{\mathrm{S}}\right)$,

where $S_{i} \equiv 2 \alpha_{i} / R$ and

$\mathcal{F}_{i}(z)=\sinh \left[\frac{S_{i}}{2}(L-z)\right] / \sinh \left[\frac{S_{i} L}{2}\right]$.

Once the effective term $b_{\text {halo }}^{\text {eff }}$ has been computed, it is possible to switch on low-energy effects and to solve Eq. (3) with all propagation processes using the usual Crank-Nicholson algorithm.

In practice, the electron source term is not a Dirac function but follows a continuum distribution in energy, which depends on the actual source considered (e.g. spallation in the disc, DM, pulsars). We therefore have to compute the pinching coefficients $\xi_{i}\left(E, E_{\mathrm{S}}\right)$ for each electron energy at source $E_{\mathrm{S}}$, which requires a very long computational time. However, an alternative way consists in averaging the quantity $\xi_{i}\left(E, E_{\mathrm{S}}\right)$ over electron energies at source $E_{\mathrm{S}}$. We show in Sect. 2.3 that the effect arising from this simplification is kept below $0.2 \%$ over the whole energy range. We describe in the following how to perform such averaging.

Let $\mathcal{P}_{i}\left(E, E_{\mathrm{S}}\right)=p_{i}\left(E, E_{\mathrm{S}}\right) \mathrm{d} E_{\mathrm{S}}$ be the probability that an electron, injected with energy in the range $\left[E_{\mathrm{S}}, E_{\mathrm{S}}+\mathrm{d} E_{\mathrm{S}}\right]$ and measured at the Earth with an energy $E$, contributes to the $i$ th Bessel order of the Bessel transform $P_{i}^{h}(E, 0)$. The associated probability density $p_{i}$ is then given by

$$
p_{i}\left(E, E_{\mathrm{S}}\right)=\frac{B_{i}\left(E, E_{\mathrm{S}}\right)}{\int_{E}^{+\infty} \mathrm{d} E_{\mathrm{S}} B_{i}\left(E, E_{\mathrm{S}}\right)} .
$$

Therefore, the mean value of the pinching coefficients $\xi_{i}\left(E, E_{\mathrm{S}}\right)$ is given by the expression

$\bar{\xi}_{i}(E)=\frac{\int_{E}^{+\infty} \mathrm{d} E_{\mathrm{S}}\left[J_{i}\left(E_{\mathrm{S}}\right)+4 k_{i}^{2} \int_{E}^{E_{\mathrm{S}}} \mathrm{d} E^{\prime} \frac{K\left(E^{\prime}\right)}{b\left(E^{\prime}\right)} B_{i}\left(E^{\prime}, E_{\mathrm{S}}\right)\right]}{\int_{E}^{+\infty} \mathrm{d} E_{\mathrm{S}} B_{i}\left(E, E_{\mathrm{S}}\right)}$,

and

$\bar{\xi}(E, r)=\frac{1}{\psi^{h}(E, r, 0)} \sum_{i=1}^{+\infty} J_{0}\left(\alpha_{i} \frac{r}{R}\right) \bar{\xi}_{i}(E) P_{i}(E, 0)$.

The mean pinching factor $\bar{\xi}(E)$ of secondary positrons is represented in Fig. 1 for the MIN, MED, and MAX sets of propagation parameters. As expected the pinching factor is larger in the case of MAX, that corresponds to the larger value of $K_{0}$ and $L$, where the effect of the pinching must be more important.

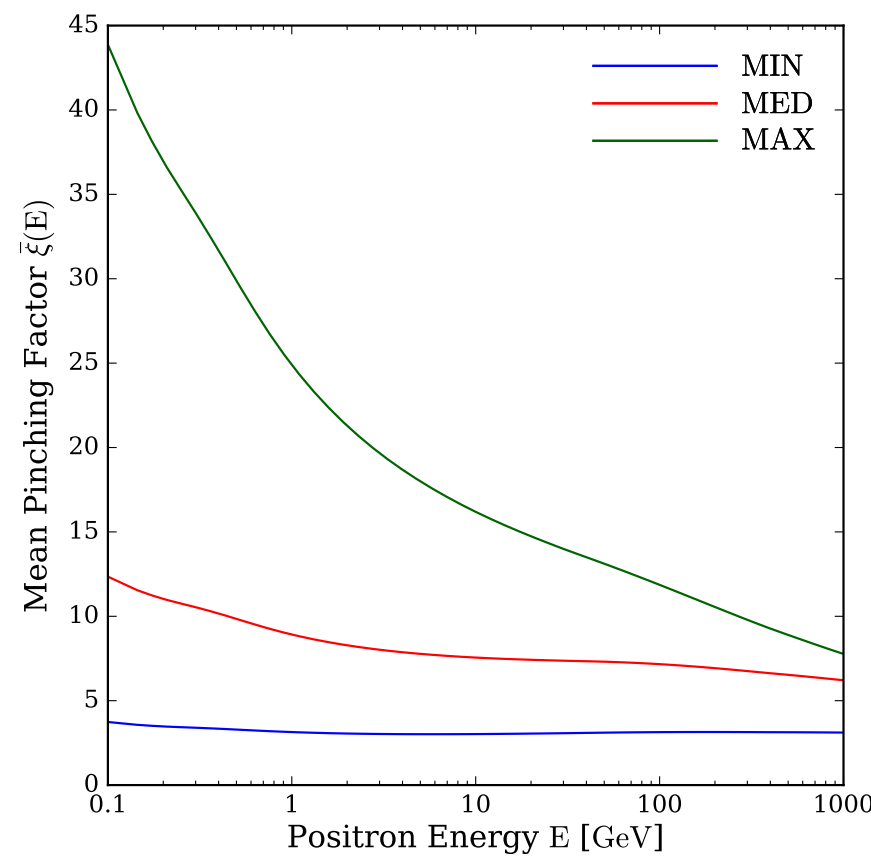

Fig. 1. Mean pinching factor of secondary positrons computed for the MIN (blue), MED (red), and MAX (green) models as a function of the positron energy.

\subsection{Testing the pinching method}

We wish to assess the theoretical uncertainty of the pinching method used to compute the positron flux. We focus our study on the energy range probed by AMS-02, that is, the rough interval [100 MeV, $1 \mathrm{TeV}]$. To this aim, we compare the analytical solution of Eq. (4) to the semi-analytical solution arising from the pinching method Eq. (13). Thus, we switch off the low-energy processes (DR, disc energy losses, convection, and destruction) and consider only halo energy losses and space diffusion processes (high-energy approximation).

We represent in the left panel of Fig. 2 the secondary positron flux at the Earth computed in the high-energy approximation scheme with the MED model. The red solid line represents the analytical solution whereas the blue dotted line represents the semi-analytical solution obtained when IC scattering and synchrotron energy losses are pinched in the Galactic disc. The relative error arising from the pinching method is shown in the right panel of Fig. 2 for MIN (blue), MED (red), and MAX (green). Furthermore, we plot in the left panels of Figs. 3 and 4 both solutions for the primary positron flux produced by a $350 \mathrm{GeV}$ DM particle annihilating into $\mu^{+} \mu^{-}$and a $1 \mathrm{TeV}$ DM particle annihilating into $b \bar{b}$, respectively. The cross-section is taken to be $\langle\sigma v\rangle=3 \times 10^{-26} \mathrm{~cm}^{3} \mathrm{~s}^{-1}$. The relative error corresponding is represented in the right panels of Figs. 3 and 4.

For secondary positrons, this error is always kept below $0.1 \%$. Our method is therefore very accurate at computing positrons produced by $p$ and He spallation onto the ISM. Regarding the primary contribution from DM annihilations, as long as the positron energy is well under the DM particle mass $m_{\chi}$, the error is also very small, always below $0.2 \%$. Close to $m_{\chi}$, the steep decrease of the positron flux (which eventually vanishes at $E \geq m_{\chi}$ ) induces a fast increase of the relative error. However, the error is above $0.2 \%$ only for energies at which the positron 

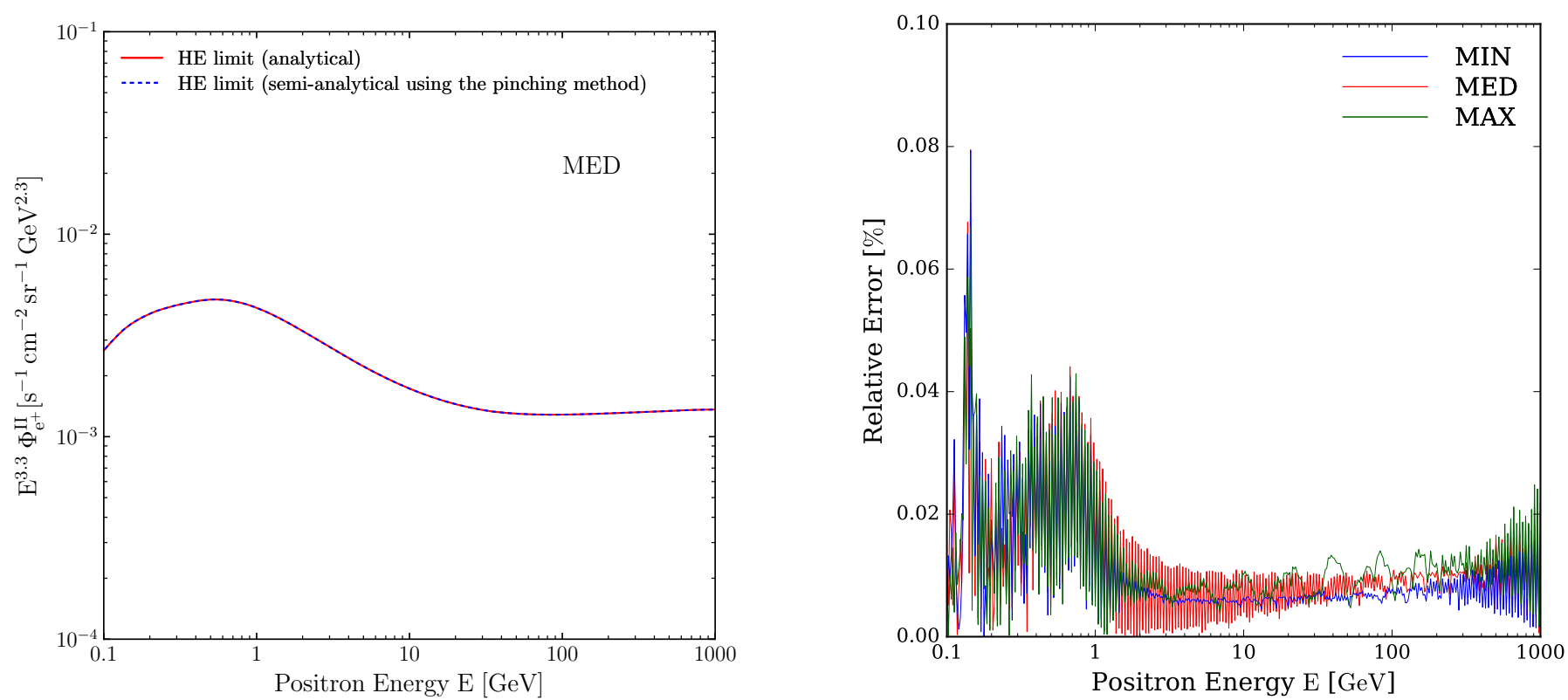

Fig. 2. Left panel: IS secondary positron flux (multiplied by $E^{3.3}$ ) in the high-energy approximation scheme for the MED model. Right panel: relative error using the pinching method for secondary positrons.
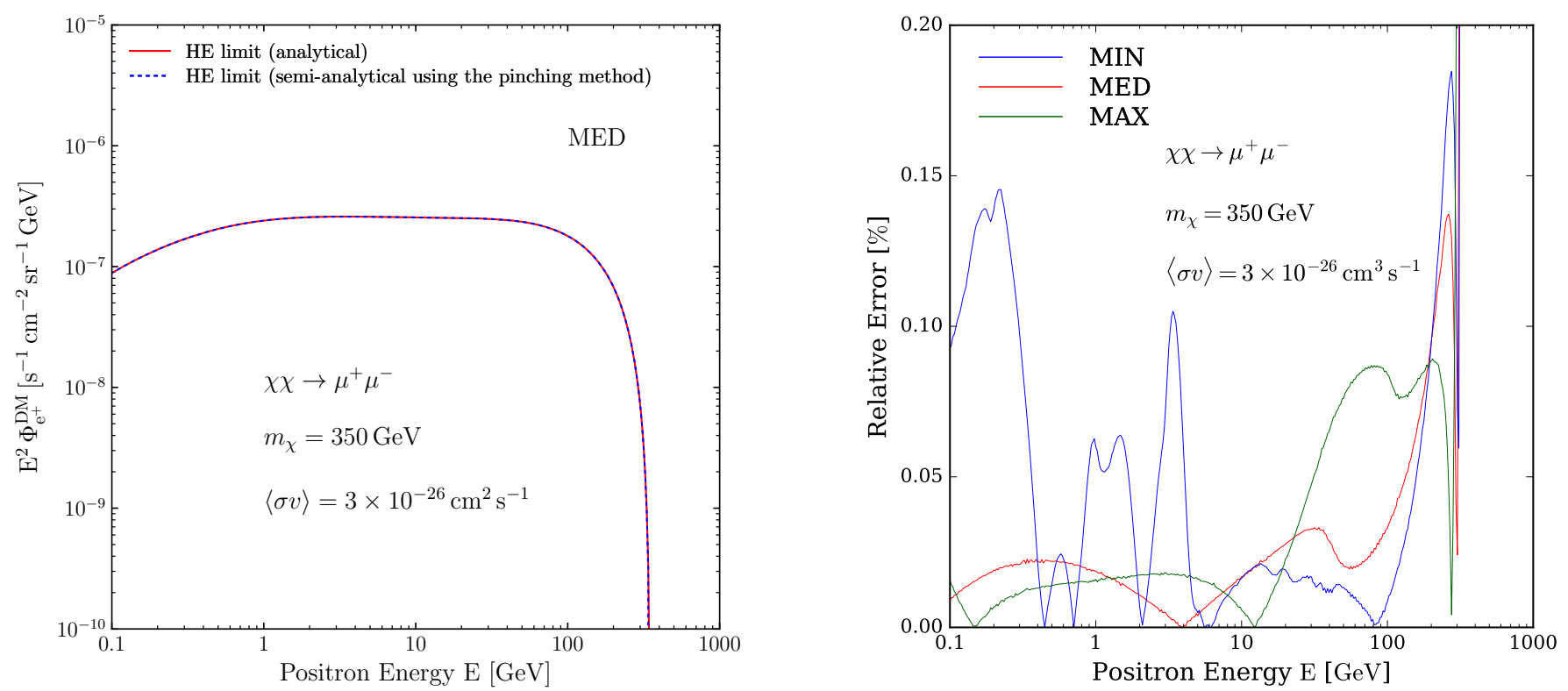

Fig. 3. Left panel: IS positron flux (multiplied by $E^{2}$ ) produced by a $350 \mathrm{GeV}$ DM particle annihilating into $\mu^{+} \mu^{-}$pairs with $\langle\sigma v\rangle=3 \times 10^{-26} \mathrm{~cm}^{3} \mathrm{~s}^{-1}$ in the high-energy approximation scheme for the MED model. Right panel: relative error using the pinching method.

flux is highly suppressed. Therefore, we can safely consider that our technique will not introduce any sizeable bias in the analysis.

Given its generality, expression (22) enables us to pinch IC and synchrotron energy losses in the Galactic disc regardless of their origins, that is, whether they are secondary or primary CRs. Thus, we can predict for the first time the electron flux at the Earth, including all propagation effects, using the semianalytical resolution of the transport equation. In the following sections, we will apply our method to both secondary and primary CRs from DM annihilation to illustrate important differences with previous treatment. Our goal is now to recompute in the most accurate way propagation constraints from positron flux at the Earth and then reinvestigate the DM explanation of the excess.

\section{Implications for secondary positrons and the dark matter signal}

In this section, we compute the source term of secondary positrons with the up-to-date primary proton and helium fluxes. The interstellar flux of secondary positrons is derived semianalytically with the pinching method presented in Sect. 2. We then focus on the DM signal coming, as an illustration, from a $10 \mathrm{TeV}$ WIMP annihilating into $\bar{b} b$ quark pairs. These secondary and primary fluxes, computed including all the propagation processes, are compared with the ones derived from the highenergy approximation. 
M. Boudaud et al.: The pinching method for Galactic cosmic ray positrons: Implications in the light of precision measurements
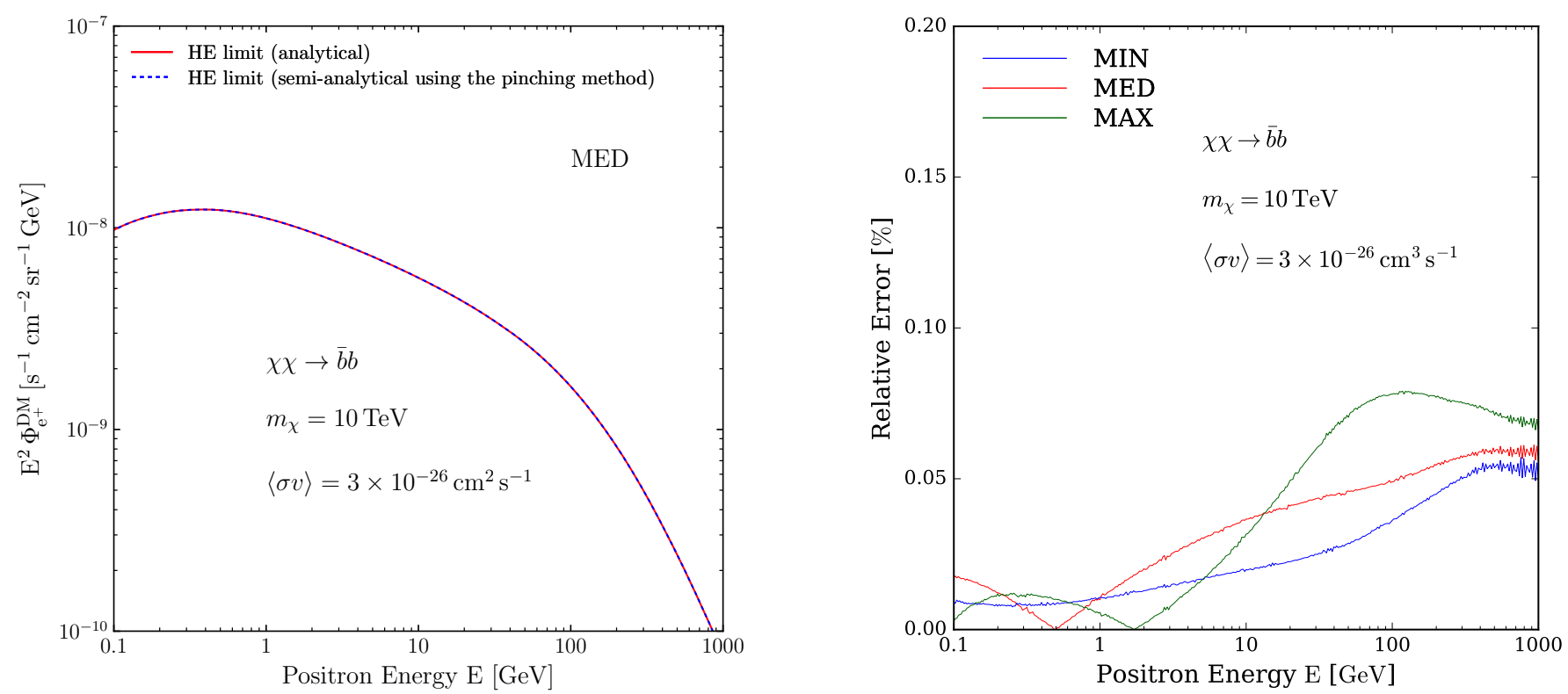

Fig. 4. Same as Fig. 3 with a $1 \mathrm{TeV}$ DM particle annihilating into $b \bar{b}$ pairs.
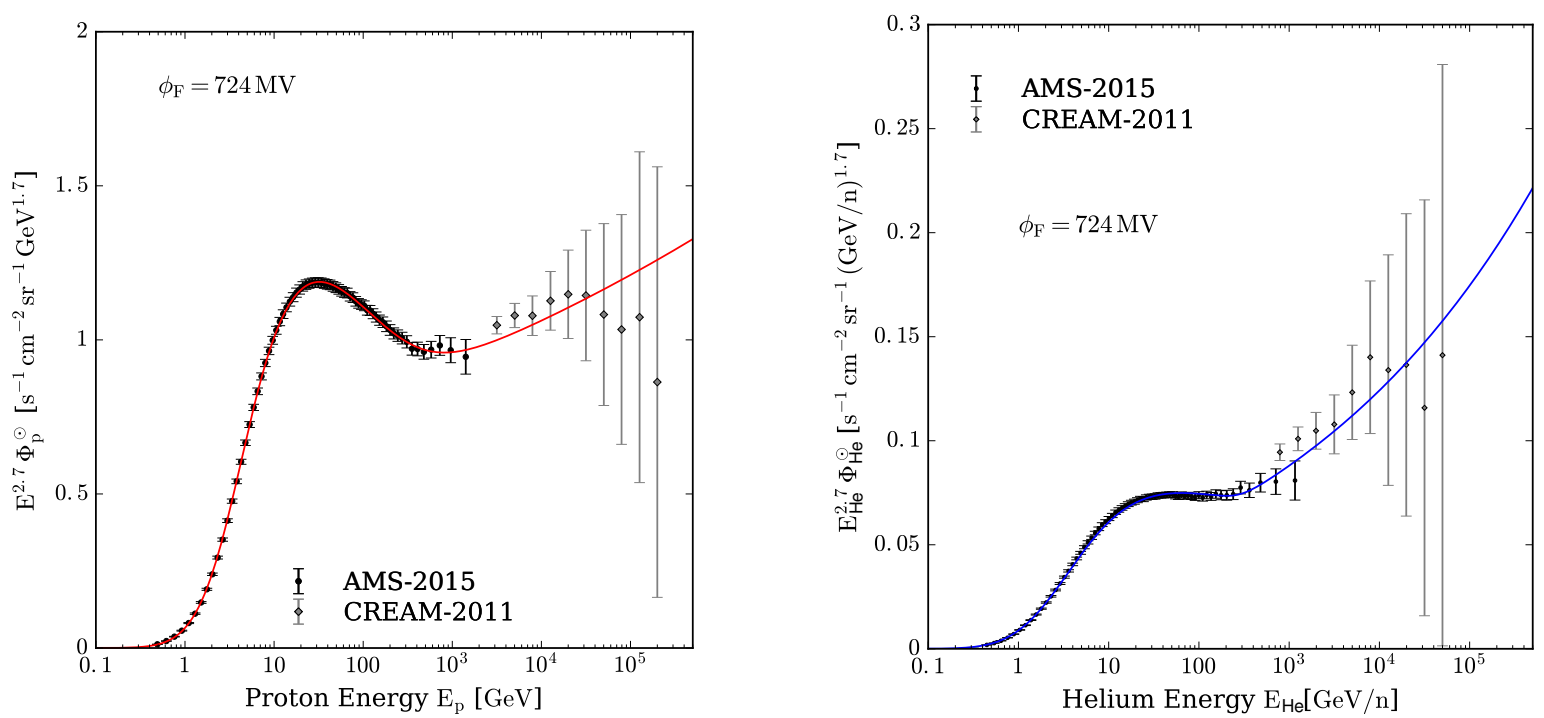

Fig. 5. Proton (left panel) and helium (right panel) fluxes (multiplied by $E^{2.7}$ ) as a function of kinetic energy for AMs-02 (Aguilar et al. 2015b,a) and CREAM (Yoon et al. 2011) data. The red and blue curves are the fitted proton and helium fluxes corresponding to Eqs. (24) and (25), respectively. We use the value from Ghelfi et al. (2016) of 724 MV for the Fisk potential $\phi_{\mathrm{F}}$.

\subsection{Astrophysical background of secondary positrons}

Secondary positrons originate from the decay of pions, kaons, and delta baryons produced by inelastic collisions of primary CR protons and helium nuclei on the ISM. The injection rate of secondary positrons reads:

$Q^{\mathrm{II}}(E, r, z)=4 \pi \sum_{j=p, \mathrm{He}} \sum_{i=\mathrm{H}, \mathrm{He}} n_{i} \int \mathrm{d} E_{j} \frac{\mathrm{d} \sigma_{j i}}{\mathrm{~d} E}\left(E_{j} \rightarrow E\right) \Phi_{j}\left(E_{j}, r, z\right)$,

where $n_{i}$ labels the atomic density of the nucleus $i$ in the ISM, $\mathrm{d} \sigma_{j i} / \mathrm{d} E$ indicates the positron differential production crosssection, and $\Phi_{j}$ stands for the CR proton and helium fluxes. We use the parameterisation of the proton-proton interaction differential cross-section derived by Kamae et al. (2006). For proton-helium interactions, we take the parameterisation from
Norbury \& Townsend (2007). To obtain the proton and helium fluxes everywhere in the Galaxy, we apply the retropropagation method introduced by Maurin et al. (2001), which requires the TOA flux as an input. This work is based on the latest measurements by AMs-02 (Aguilar et al. 2015b,a) and CREAM (Yoon et al. 2011). The proton and helium fluxes are fitted using a model introduced in Aguilar et al. (2015b,a), where a single power law in rigidity $R^{\gamma}$ exhibits a smooth transition to $R^{\gamma+\Delta \gamma}$ above the rigidity $R_{\mathrm{b}}$. The smoothness of the spectral index transition is described by the parameter $s$. An additional effective parameter $\alpha$ is used to fit the low-rigidity part of the proton flux. The interstellar (IS) primary fluxes can be described as follows:

$\Phi_{p}^{\mathrm{IS}}(R)=C \beta\left(1-\mathrm{e}^{\alpha R}\right) R^{\gamma}\left[1+\left(\frac{R}{R_{\mathrm{b}}}\right)^{\Delta \gamma / s}\right]^{S}$, 
Table 3. Typical values of the relative error $\left(\Phi_{\mathrm{HE}}^{\mathrm{II}}-\Phi^{\mathrm{II}}\right) / \Phi^{\mathrm{II}}(\%)$ of the high-energy approximation for secondary positrons compared to the exact result.

\begin{tabular}{cccccc}
\hline \hline Positron energy $(\mathrm{GeV})$ & 10 & 50 & 100 & 500 & 1000 \\
\hline MIN & 48 & 17 & 9.7 & 2.5 & 1.4 \\
MED & 19 & 7.7 & 4.7 & 1.4 & 0.8 \\
MAX & 1.7 & 2.0 & 1.5 & 0.6 & 0.4 \\
\hline
\end{tabular}

and

$\Phi_{\mathrm{He}}^{\mathrm{IS}}(R)=C \beta R^{\gamma}\left[1+\left(\frac{R}{R_{\mathrm{b}}}\right)^{\Delta \gamma / s}\right]^{S}$,

with $\beta$ being the particle velocity. The force-field approximation (Fisk 1971) is used to obtain the relation between $\Phi_{\text {IS }}$ and $\Phi_{\mathrm{TOA}}$, that is, the IS and TOA fluxes, respectively. The value $\phi_{\mathrm{F}}=724 \mathrm{MV}$ determined by Ghelfi et al. (2016) is used hereafter unless explicitly stated. This model has been fitted to the measured fluxes, as is shown in Fig. 5, yielding the parameter values reported in Table 2 .

The interstellar flux of secondary positrons, computed with the pinching method including all propagation effects, is represented in the left panel of Fig. 6 by the solid lines for MIN (blue), MED (red), and MAX (green). The high-energy approximation, where only diffusion and halo energy losses are taken into account, is featured by the dotted lines. It is henceforth possible to assess the error made when applying the high-energy approximation often used in the literature to compute the positron flux above $10 \mathrm{GeV}$. This error is defined as $\left(\Phi_{\mathrm{HE}}^{\mathrm{II}}-\Phi^{\mathrm{II}}\right) / \Phi^{\mathrm{II}}$ where the index HE stands for high energy. This quantity is plotted in the right panel of Fig. 6, and a few numerical values are displayed in Table 3. As already noticed by Delahaye et al. (2009), the highenergy approximation tends to largely underestimate the amount of positrons below $5 \mathrm{GeV}$. Interestingly, we find on the other hand that above that value, the high-energy approximation overshoots the exact result. Indeed, although convection and disc energy losses are subdominant with respect to halo energy losses and space diffusion, they still have a sizeable effect and tend to reduce the positron flux above $10 \mathrm{GeV}$. Moreover, the relative error strongly depends on the propagation parameters, the maximum value being reached for the MIN configuration. This can be understood by the fact that the convection velocity decreases along the sequence MIN, MED, MAX. Therefore, we observe that the discrepancy with the high-energy approximation increases with higher values of the convection.

\subsection{Primary positrons from the annihilation of dark matter particles}

The source term of positrons produced by the annihilation of DM particles reads

$Q^{\mathrm{DM}}(E, \boldsymbol{x})=\eta\langle\sigma v\rangle \frac{\rho_{\chi}^{2}(\boldsymbol{x})}{m_{\chi}^{2}}\left\{\left.g(E) \equiv \sum_{i}^{N} b_{i} \frac{\mathrm{d} N}{\mathrm{~d} E}\right|_{i}\right\}$,

where $m_{\chi}$ is the DM particle mass and $\langle\sigma v\rangle$ its average annihilating cross section. The value of $\eta$ depends on whether the DM particle is Majorana-type $(\eta=1 / 2)$ or Dirac-type $(\eta=1 / 4)$. We use the DM density profile introduced by Navarro et al. (1997), hereafter denoted NFW, with the local DM density $\rho_{\odot}=$ $0.3 \mathrm{GeV} \mathrm{cm}^{-3}$ (Bovy \& Tremaine 2012). The energy distribution of positrons $g(E)$ at the source is obtained by summing over
Table 4. Typical values of the relative error $\left(\Phi_{\mathrm{HE}}^{\mathrm{DM}}-\Phi^{\mathrm{DM}}\right) / \Phi^{\mathrm{DM}}$ of the high-energy approximation for primary positrons compared to the exact result.

\begin{tabular}{cccccc}
\hline \hline Positron energy $(\mathrm{GeV})$ & 10 & 50 & 100 & 500 & 1000 \\
\hline MIN & 74 & 18 & 10 & 2.6 & 1.4 \\
MED & 22 & 6.2 & 3.8 & 1.1 & 0.6 \\
MAX & 4.4 & 1.3 & 0.7 & 0.5 & 0.4 \\
\hline
\end{tabular}

the individual contributions $\mathrm{d} N /\left.\mathrm{d} E\right|_{i}$ for each annihilating channel $i$ weighted by the branching ratio $b_{i}$. The individual energy distributions $\mathrm{d} N /\left.\mathrm{d} E\right|_{i}$ are computed with the micrOMEGAs_3.6 package (Bélanger et al. 2011, 2014).

For illustrative purposes, we consider throughout this section a Majorana-type DM species with a mass $m_{\chi}$ of $10 \mathrm{TeV}$ annihilating into $\bar{b} b$ quark pairs with the thermal cross section $\langle\sigma v\rangle=3 \times 10^{-26} \mathrm{~cm}^{3} \mathrm{~s}^{-1}$. The IS flux of primary positrons computed with all propagation processes taken into account is featured by the solid lines in the left panel of Fig. 7. The highenergy approximation corresponds to the dotted lines. The relative error $\left(\Phi_{\mathrm{HE}}^{\mathrm{DM}}-\Phi^{\mathrm{DM}}\right) / \Phi^{\mathrm{DM}}$ is plotted in the right panel whereas a few numerical values are displayed in Table 4 . We notice differences in the magnitude of this error, depending on the CR propagation configuration. We attribute them to the different values of the convective velocity $V_{\mathrm{c}}$. Actually, positrons produced by $\mathrm{DM}$ annihilating throughout the $\mathrm{MH}$ are more sensitive to convection than secondary positrons, which originate from the Galactic disc. As a consequence, the error associated to the highenergy approximation tends to be larger for primary positrons than for secondary ones. In the former case, it is significantly large in the MIN model for which $V_{\mathrm{c}}$ is the highest.

In summary, we have computed the flux of positrons including all the propagation effects for the secondary component as well as the DM signal. We have shown that low-energy effects modify drastically the shape of the positron spectrum. In addition, these effects could have a sizeable importance above $10 \mathrm{GeV}$, in contrast to what has been assumed in the literature. At $10 \mathrm{GeV}$, they modify the prediction up to $48 \%$ for the secondary component, and up to $74 \%$ for the DM signal, in the MIN configuration. Therefore, neglecting the low-energy CR propagation processes could lead to misleading interpretations when attempting to compare the theoretical predictions to the highaccuracy data provided by the AMS- 02 collaboration. All the results presented in the following of this paper are obtained using the pinching method to solve the full transport equation Eq. (1).

\section{Constraining propagation parameters with AMs-02 data}

\subsection{Secondary positrons and propagation models}

Secondary cosmic rays are often regarded as a powerful observable to constrain the propagation scenario. Instead of relying on unknown source modelisation, their source term is determined by primary particles, for which precise measurements are available, therefore allowing one to more easily disentangle propagation from injection effects ${ }^{1}$. This is the case of the boron flux, commonly divided by the carbon flux, so that the $\mathrm{B} / \mathrm{C}$ ratio no longer depends on the carbon injection assumptions. Secondary isotopes of helium and hydrogen (see for example Coste et al. 2012) and subFe/Fe ratio are also used for that

\footnotetext{
1 Potential contamination by non-negligible primary component could spoil such ability, see e.g. Genolini et al. (2015) for a discussion.
} 
M. Boudaud et al.: The pinching method for Galactic cosmic ray positrons: Implications in the light of precision measurements
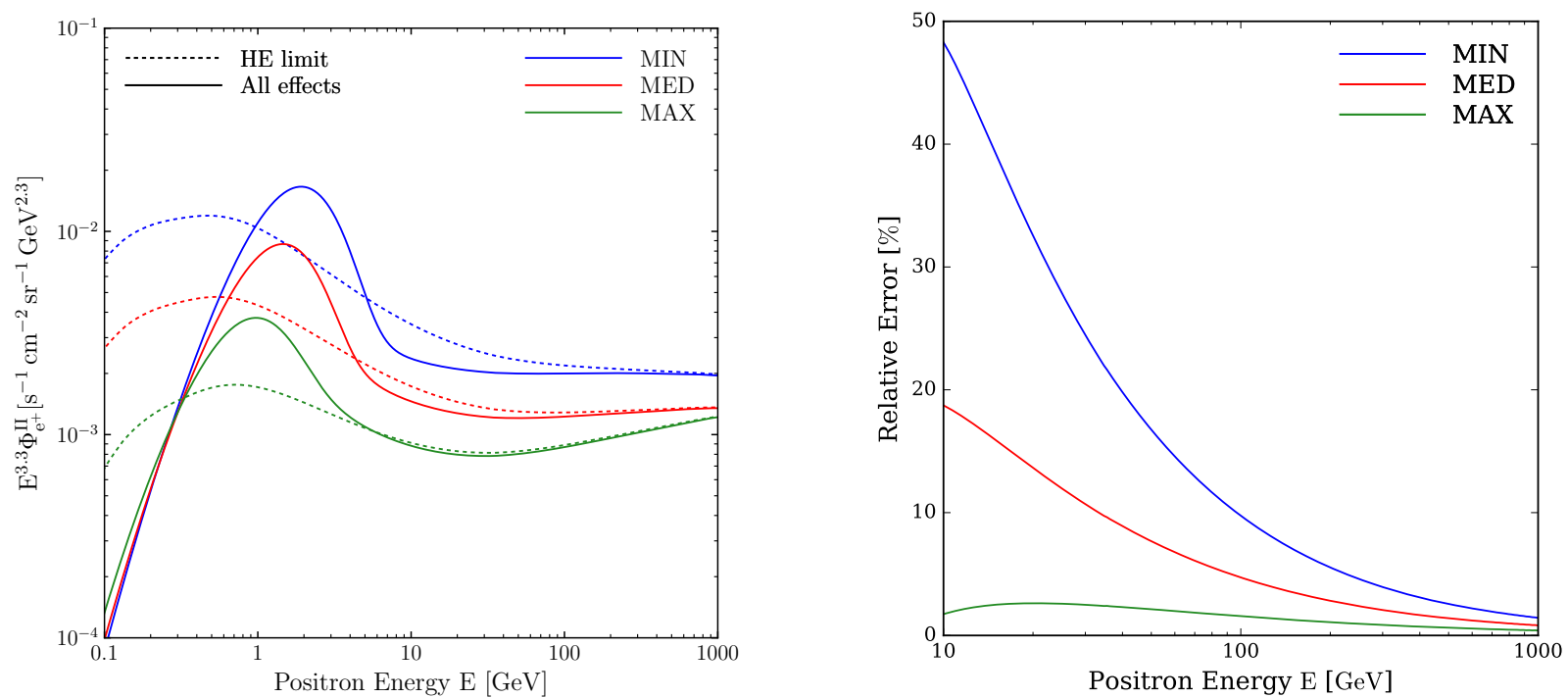

Fig. 6. Left panel: interstellar flux (multiplied by $\left.E^{3.3}\right)$ of secondary positrons computed with all propagation effects $\left(\Phi^{\mathrm{II}}\right.$, solid lines) and with the high-energy approximation $\left(\Phi_{\mathrm{HE}}^{\mathrm{II}}\right.$, dotted lines) for the MIN (blue), MED (red), and MAX (green) models. Right panel: relative error $\left(\Phi_{\mathrm{HE}}^{\mathrm{II}}-\Phi^{\mathrm{II}}\right) / \Phi^{\mathrm{II}}$ above $10 \mathrm{GeV}$ of the high-energy approximation for secondary positrons compared to the exact result.

Table 2. Values of the proton and helium flux parameters resulting from a fit to the AMs-02 (Aguilar et al. 2015b,a) and CREAM (Yoon et al. 2011) data assuming $\phi_{\mathrm{F}}=724 \mathrm{MV}$.

\begin{tabular}{ccccccc}
\hline \hline & $C\left[\mathrm{~m}^{-2} \mathrm{~s}^{-1} \mathrm{sr}^{-1} \mathrm{GV}^{-1}\right]$ & $\alpha\left[\mathrm{GV}^{-1}\right]$ & $\gamma$ & $R_{\mathrm{b}}[\mathrm{GV}]$ & $\Delta \gamma$ & $s$ \\
\hline Proton & $(2.71 \pm 0.02) \times 10^{4}$ & $-0.512 \pm 0.012$ & $-2.88 \pm 0.01$ & $424 \pm 158$ & $0.242 \pm 0.056$ & $0.156 \pm 0.072$ \\
\hline Helium & $(3.56 \pm 0.04) \times 10^{3}$ & - & $-2.77 \pm 0.01$ & $543 \pm 163$ & $0.213 \pm 0.045$ & $0.047 \pm 0.018$ \\
\hline
\end{tabular}
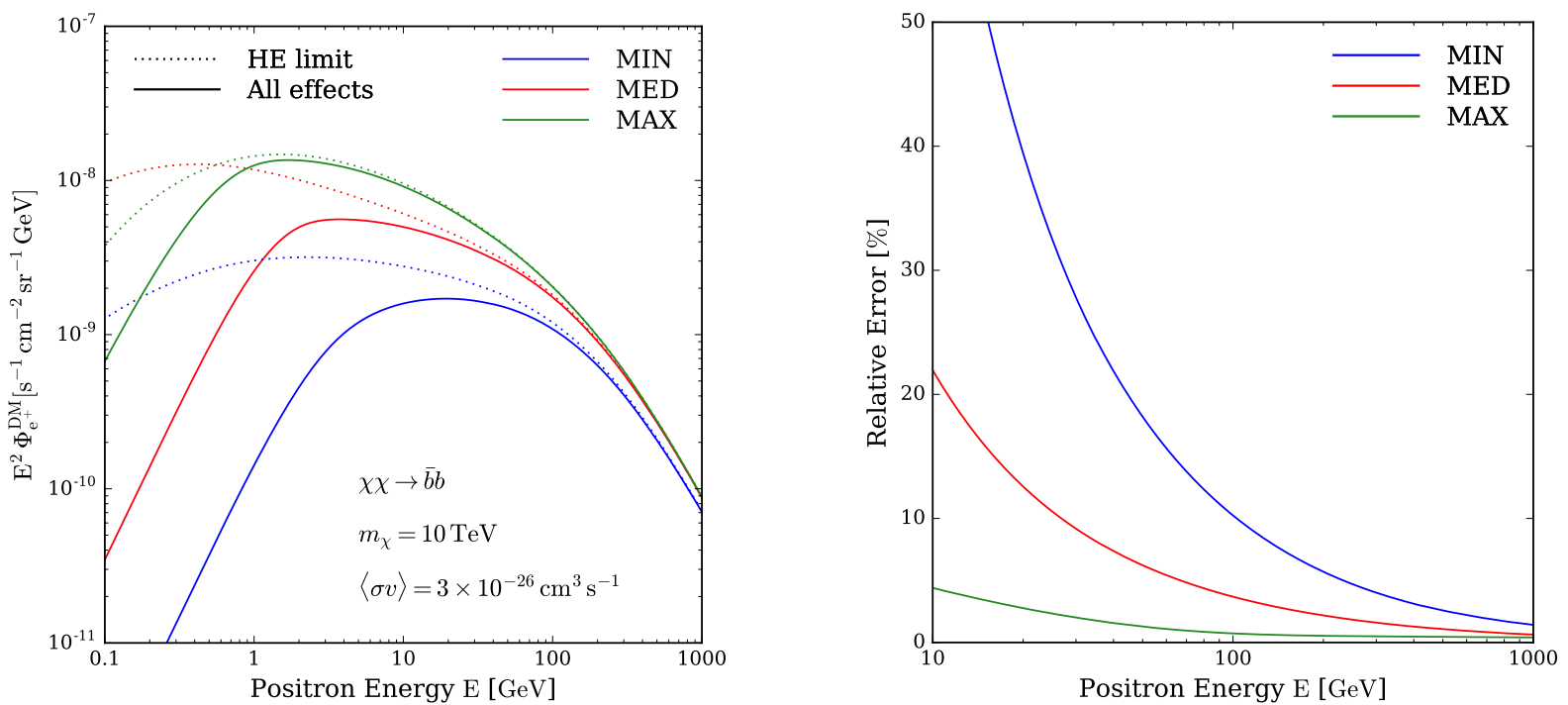

Fig. 7. Left panel: interstellar flux (multiplied by $E^{2}$ ) of primary positrons computed with all propagation effects $\left(\Phi^{\mathrm{DM}}\right.$, solid lines) and with the high-energy approximation ( $\Phi_{\mathrm{HE}}^{\mathrm{DM}}$, dotted lines) for a $10 \mathrm{TeV}$ WIMP annihilating into $\bar{b} b$ pairs with $\langle\sigma v\rangle=3 \times 10^{-26} \mathrm{~cm}^{3} \mathrm{~s}^{-1}$, for the MIN (blue), MED (red), and MAX (green) models. Right panel: relative error $\left(\Phi_{\mathrm{HE}}^{\mathrm{DM}}-\Phi^{\mathrm{DM}}\right) / \Phi^{\mathrm{DM}}$ above $10 \mathrm{GeV}$ of the high-energy approximation for primary positrons compared to the exact result.

purpose, and lead to similar understanding of CR propagation in our Galaxy.

Until the discovery of a high-energy excess, positrons were thought for a long time as being purely secondary particles. Although its secondary component tends to be forgotten after the excitement of such a discovery, it still carries a wealth of information on propagation properties. In fact, as noticed in
Lavalle et al. (2014), in many propagation models compatible with the $\mathrm{B} / \mathrm{C}$ ratio (especially those with a small halo size), pure secondary predictions of the flux at the lowest energies (typically below $4 \mathrm{GeV}$ ) are not in deficit but rather in excess with respect to measurements. This observation has been shown to yield a useful complementary constraint on the propagation parameters. Indeed, since the flux of secondary positrons scales 

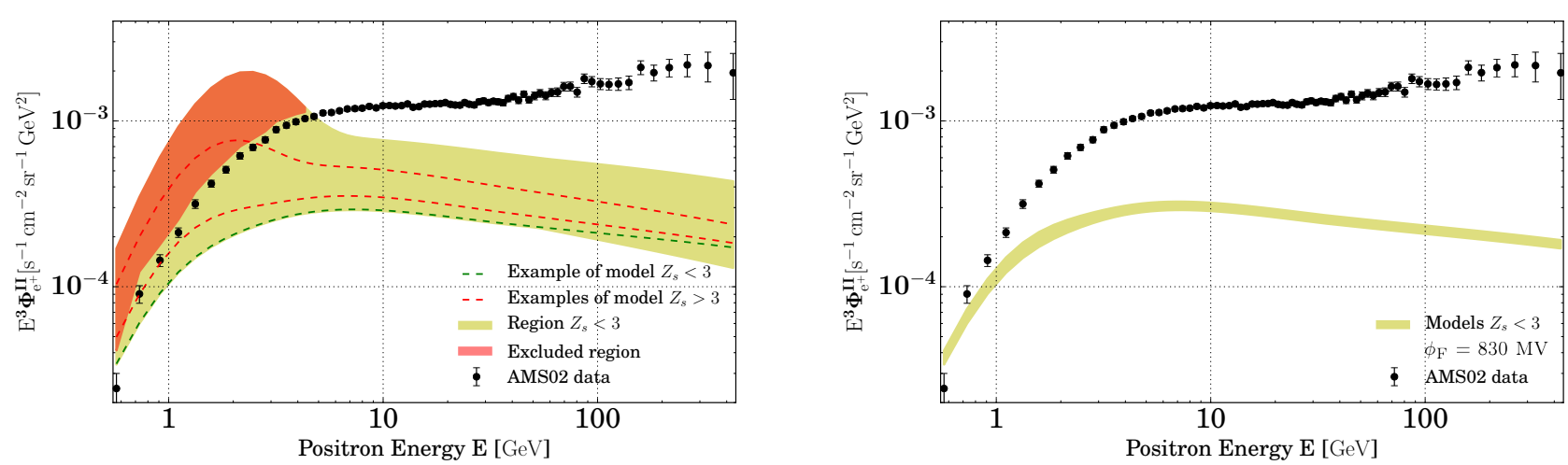

Fig. 8. Left panel: positron flux (multiplied by $E^{3}$ ) from the AMS-02 data compared to the envelope of the 1,623 flux predictions for the secondary positrons (coloured band). The red-coloured region is the domain that is crossed by all the excluded models. The dashed red lines show two examples of models that do not fulfill the constraints $Z_{i}<3$ for all energy bins $i$ (i.e. the Z-score constraint defined as $Z_{\mathrm{s}}=\max \left(Z_{i}\right)<3$ ). The dashed green line represents an example of a model that fulfills the constraints $Z_{i}<3$ for all energy bins $i$. Right panel: positron flux (multiplied by $E^{3}$ ) from the AMS-02 data compared to the envelope of the 54 models fulfilling $Z_{i}<3$ for all energy bins $i$.

as the ratio of the production volume over the diffusion volume, leading to $1 / \sqrt{K_{0}}$ dependency, the well-known degeneracy $K_{0} / L$ introduced by secondary-to-primary ratio studies can be lifted. However, in order to use this complementarity, one needs in practice to be able to accurately compute the positron spectrum at the lowest energies, despite the presence of DR, convection, and disc energy losses. In Lavalle et al. (2014), a qualitative trick was used; it was argued that the inclusion of DR would lead to the formation of a bump around $1 \mathrm{GeV}$, which tends to increase the flux with respect to cases in which it is neglected, thus leading to a predicted flux in excess of the data. We have shown in Sect. 3 that the competition between DR, convection, and disc energy losses tends to the formation of such a bump around $2 \mathrm{GeV}$. However, already above $5 \mathrm{GeV}$, there might be parts of the $\left\{V_{\mathrm{c}}, V_{\mathrm{a}}\right\}$ parameter space that actually lead to a decrease of the flux. This is particularly pronounced in the MIN model as shown in Fig. 6. We will therefore recompute the constraints of Lavalle et al. (2014) with i) our full-resolution method at low energy; and ii) updated fluxes measured by AMs-02. This will lead to more robust and more stringent constraints on the propagation parameters.

\subsection{Skimming method for the propagation models}

We compute the secondary positron flux for the 1623 propagation parameter sets selected by the $\mathrm{B} / \mathrm{C}$ ratio analysis of Maurin et al. (2001). These parameters are sorted from a uniform linear grid in the propagation parameter space, namely $(\delta$, $\left.K_{0}, L, V_{\mathrm{c}}, V_{\mathrm{a}}\right)$, and are in agreement with the HEA03 $\mathrm{B} / \mathrm{C}$ ratio within three standard deviations. The secondary positrons are calculated including all the effects described in Sect. 2 and recalled hereafter: diffusion, convection, reacceleration, highenergy losses (IC, synchrotron), low-energy losses (adiabatic, ionisation, coulombic, bremsstrahlung), retro-propagation of the proton and helium fluxes, annihilation, and solar modulation. One may worry that our constraints highly depend on solar modulation modelisation. Although no extensive study of solar modulation for positrons during the period for which AMS-02 has been taking data is available, this modulation is commonly assumed to affect equally particles of the same rigidity and the same sign of charge. This assumption will soon be tested by the forthcoming AMS- 02 measurements of the variations of the positron-to-proton ratio over the last solar cycle. Therefore, within the force-field approximation, we can rely on studies of the proton solar modulation such as in Ghelfi et al. (2016) and make use of the Fisk potential derived there. In a conservative approach, we lower the secondary prediction as much as possible using the $3 \sigma$ highest Fisk potential, which was found by Ghelfi et al. (2016) to be 830 MV. The constraints derived with this high value might not be optimal. They already provide relatively strong conclusions as discussed in the following sections.

In order to quantify, for a given propagation model, the deviations of the predicted flux from the data, and any potential overshooting, we follow the criterion advocated in Lavalle et al. (2014), and first define, for each energy bin, the quantity

$Z_{i}=\frac{\Phi_{\mathrm{e}^{+}}^{\mathrm{II}}\left(E_{i}\right)-\Phi^{\mathrm{data}}\left(E_{i}\right)}{\sigma^{\mathrm{data}}\left(E_{i}\right)}$

where $\Phi_{\mathrm{e}^{+}}^{\mathrm{II}}\left(E_{i}\right)$ is the predicted secondary positron flux in a given energy bin, $\Phi^{\text {data }}\left(E_{i}\right)$ is the corresponding experimental flux, and $\sigma^{\text {data }}\left(E_{i}\right)$ its experimental uncertainty. A propagation model is allowed provided that $Z_{i}$ does not exceed 3 whatever the energy bin. In other words, for selected models, we allow predictions to overshoot the data by at most three standard deviations in each energy bin. We note that, unlike Lavalle et al. (2014), we do not combine the values of $Z_{i}$ at different energies into a single statistical test. To do so, one would need to know correlations of experimental uncertainties between different energies, but those are not provided by the AMS- 02 collaboration. One could assume uncorrelated uncertainties, but this would only be true for the statistical ones. We therefore consider bins separately, making our test a conservative choice, of which there could be room for some improvement.

\subsection{Results and discussion}

An illustration of the selection method is presented in the left panel of Fig. 8. In this figure, we display the AMs-02 positron flux and superimpose a coloured band whose edges correspond to the envelope of the 1623 predictions for the secondary positrons. The red-coloured region represents predictions that overshoot the data according to our definition and therefore contains the excluded models. On the other hand, the yellowcoloured region contains all allowed models. As an example, we display in dashed green a model that fulfills the Z-score constraint defined as $Z_{s}=\max _{E_{i} \in \text { data }}\left(Z_{i}\right)<3$, and in dashed red two models that do not respect it. The right panel of Fig. 8 illustrates 
M. Boudaud et al.: The pinching method for Galactic cosmic ray positrons: Implications in the light of precision measurements
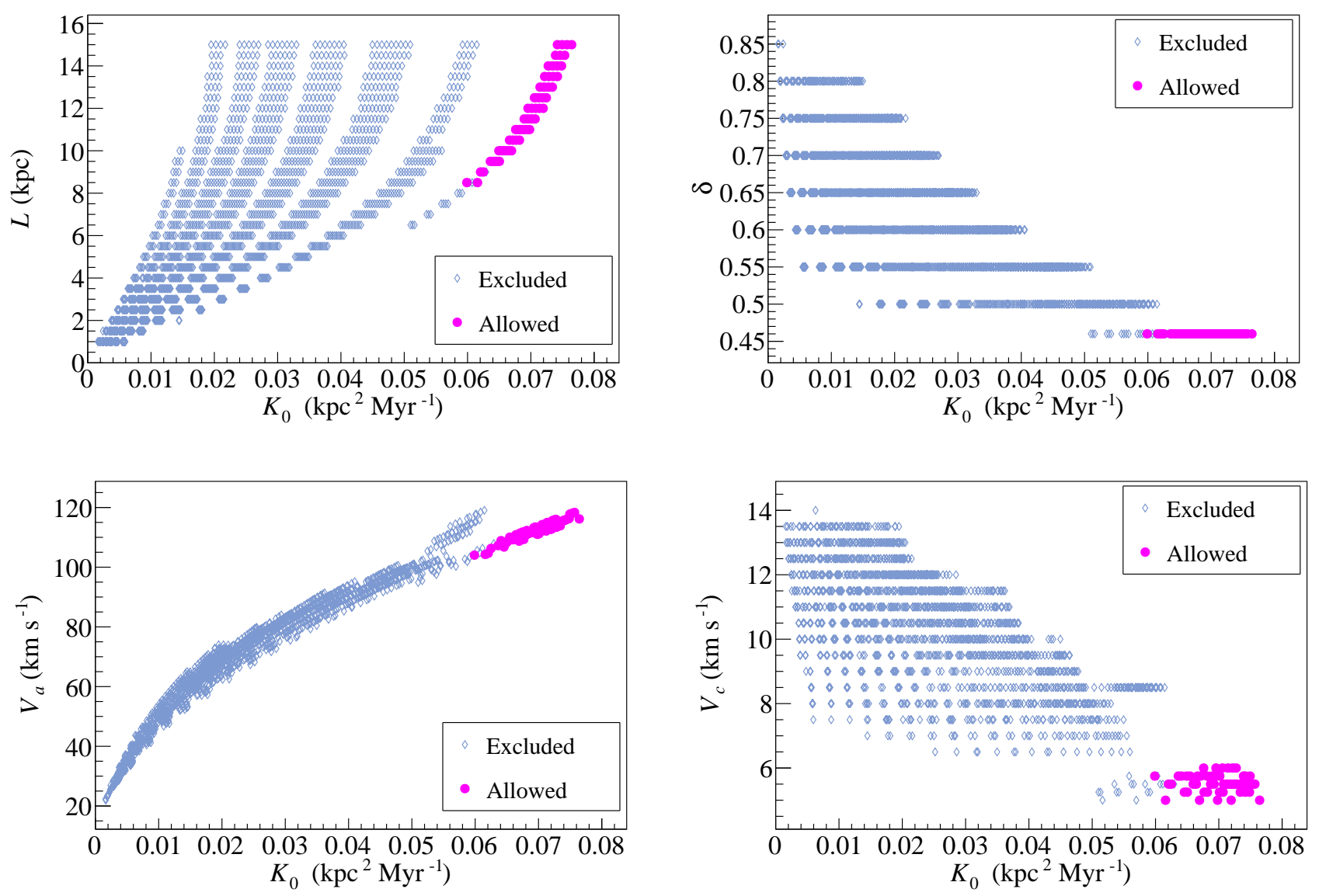

Fig. 9. Projection of the 1623 propagation parameter sets selected by the $\mathrm{B} / \mathrm{C}$ ratio (Maurin et al. 2001) in the $K_{0}-L, K_{0}-\delta, K_{0}-V_{\mathrm{a}}$, and $K_{0}-V_{\mathrm{c}}$ planes. The light blue diamonds show the propagation parameter values that are excluded, whereas the magenta circles denote the values that are allowed by this analysis.

the allowed propagation models that remain after the selection process: only 54 propagation sets out of 1623 survive the criterion. Interestingly, one can see that the positron excess measured by AMs- 02 seems to start already above $2 \mathrm{GeV}$, and not $10 \mathrm{GeV}$ as often advocated. This will reveal itself to be very complicated to explain in terms of a single primary component. Selected models are those which minimise the secondary production over the whole energy range. Figure 9 compares ranges of selected parameters with respect to their initial ones. One can see that our new method enables us to drastically reduce the allowed parameter space with respect to former $\mathrm{B} / \mathrm{C}$ analysis. Furthermore, we confirm the lifting of the degeneracy between $K_{0}$ and $L$, as one can see from the top-left panel of Fig. 9, as well as the high sensitivity to parameters that (mainly) control propagation at low energies, namely $V_{\mathrm{a}}$ and $V_{\mathrm{c}}$. Practically, common characteristics of these models are; i) a large halo size $L$ (ranging from 8.5 to $15 \mathrm{kpc}$ ) together with relatively high $K_{0}$, typically $\geq 0.06 \mathrm{kpc}^{2} \mathrm{Myr}^{-1}$; ii) a slope of the diffusion coefficient $\delta$ equal to 0.46 , the minimal value allowed by the $\mathrm{B} / \mathrm{C}$ analysis used in this study; and iii) small values of the convective wind $V_{\mathrm{c}} \leq 6 \mathrm{~km} \mathrm{~s}^{-1}$ accompanied by large values of the Alfvèn waves velocity $V_{\mathrm{a}} \geq 100 \mathrm{~km} \mathrm{~s}^{-1}$. The fact that, in our analysis, $\delta$ is confined to the edge of the range indicates that even smaller values are likely to be favoured by positron data. This affirmation is indeed confirmed since, during the writing of this article, AMs-02 published the value of $\delta=0.333 \pm 0.015$ from a power law fit of the high-rigidity pure diffusive regime of their B/C data (Aguilar et al. 2016b).
These features can be readily understood. As recalled above, the secondary positron flux scales with $1 / \sqrt{K_{0}}$. Hence, models with larger $K_{0}$ result in lower density of positrons at Earth compared to models with small diffusion coefficient. Given that secondary-to-primary ratios mostly constrain the $K_{0} / L$ ratio, selected models have a relatively high $L$, as well as a small value of $\delta$, the former being anti-correlated with $K_{0}$. Finally, values of the selected couples $\left\{V_{\mathrm{a}}, V_{\mathrm{c}}\right\}$ minimize the bump at low energies and are therefore favoured by the analysis. Interestingly, in the recent literature, models with a large halo size have been suggested by other observables. Especially, the study of the antiproton-to-proton (Aguilar et al. 2016a) and boron-tocarbon (Aguilar et al. 2016c) ratios measured by AMs-02 point as well towards a MAX-like propagation model (Giesen et al. 2015; Korsmeier \& Cuoco 2016). Radioactive species such as ${ }^{10} \mathrm{Be} /{ }^{9} \mathrm{Be}$ (Strong \& Moskalenko 2001; Putze et al. 2010) hint also at similar models although the dependence of this observable on the local density (local bubble) may bias the result. At other wavelengths (e.g. radio; Di Bernardo et al. 2013) and in diffuse gamma ray analyses (Ackermann et al. 2012), a high value of $L$ also seems to be preferred. Even more recently, it has been shown that, as far as the stochastic injection of cosmic rays is concerned, the regularity of the proton spectrum could arise from a large magnetised halo size (Genolini et al. 2017). Our results are in very good agreement with all these different observables, which therefore all underline the need for a primary positron component in order to explain data above a few $\mathrm{GeV}$. In the following section, we investigate the consequences of our 

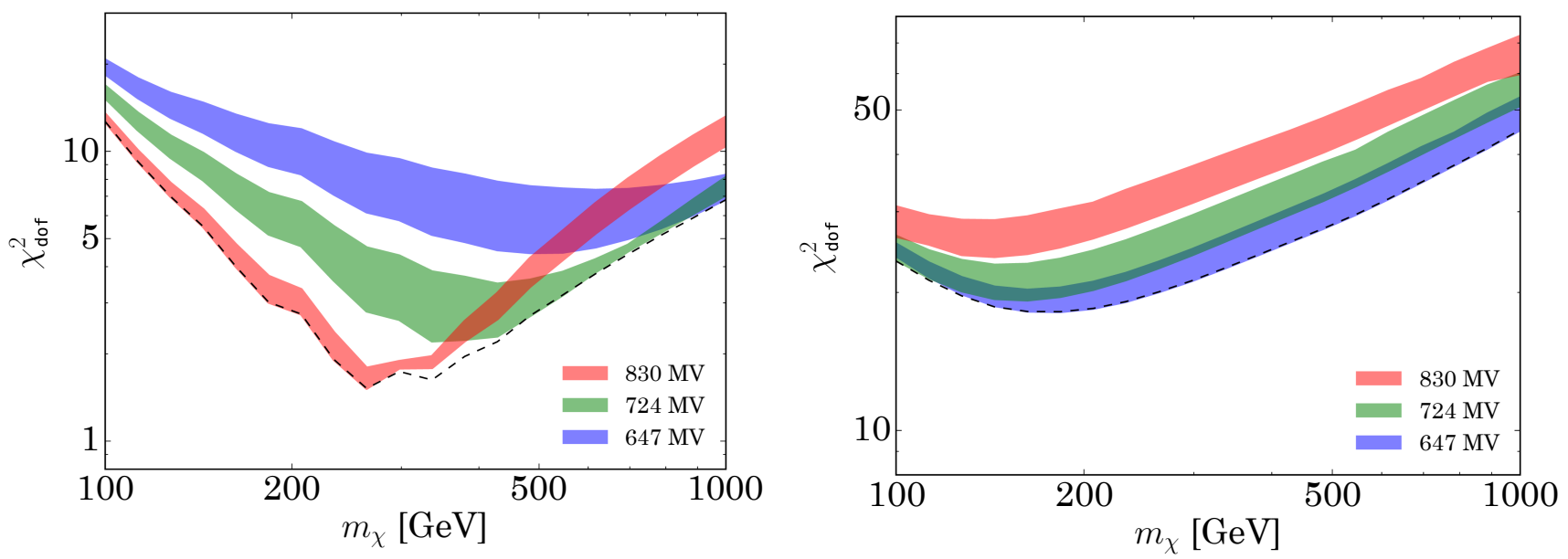

Fig. 10. Evolution of the $\chi_{\text {d.o.f }}^{2}$ as a function of the DM mass $m_{\chi}$ in the case of direct annihilation into standard model particles (left panel) and annihilation into four leptons through light mediators (right panel). The results of the analysis are displayed using a Fisk potential of $830 \mathrm{MV}, 724$ $\mathrm{MV}$, and $647 \mathrm{MV}$ in red, green, and blue, respectively. The black dashed line represents the minimal $\chi_{\text {dof }}^{2}$ among the seven Fisk potentials and 54 propagation models. The thickness of the coloured band is obtained by scanning over the 54 propagation models.

updated propagation constraints on the hypothesis of dark matter annihilations as the source of this primary component.

\section{Dark matter interpretation of the Ams-02 data}

\subsection{Dark matter fitting procedure}

The most striking feature of the positron flux data is the highenergy gap with respect to the secondary prediction. Filling this gap with a dark matter component has been the concern of many studies, but semi-analytical methods were always restricted above $10 \mathrm{GeV}$ (see for example Boudaud et al. 2015). Hereafter, we use the resolution method of Sect. 2 to compute the positron flux following dark matter annihilation over the whole energy range covered by AMs-02 data. Due to the important statistics of data below $10 \mathrm{GeV}$, constraints based only on the quality of the fit become more stringent.

Technically, we use the 54 propagation models selected in Sect. 4 to compute the theoretical prediction of the positron flux, which is the sum of a primary component coming from dark matter annihilation and the secondary component,

$\Phi_{\mathrm{e}^{+}}^{\mathrm{th}}=\Phi_{\mathrm{e}^{+}}^{\mathrm{DM}}+\Phi_{\mathrm{e}^{+}}^{\mathrm{II}}$.

We consider two different cases: DM particle annihilating into a general final state composed of quarks, leptons, and bosons, and the case of a leptophilic DM that annihilates into a combination of leptonic channels through a light mediator.

In a similar vein as Boudaud et al. (2015), we make no assumptions about the underlying DM model and consider the possibility that DM annihilates into a combination of channels, namely $b \bar{b}, W^{+} W^{-}, \mathrm{e}^{+} \mathrm{e}^{-}, \mu^{+} \mu^{-}$, and $\tau^{+} \tau^{-}$, with a branching ratio free to vary. The limited choice of these channels relies on the fact that they describe relatively well the various spectrum shape, and avoids introducing too many free parameters. For example, the $b \bar{b}$ channel typically describes the spectra of the different quark and gluon final states. To a certain extent positron spectra following Higgs decay are also similar to the $b \bar{b}$ case, since the Higgs decays dominantly into hadrons. Finally, the $W^{+} W^{-}$ channel is chosen to describe positron spectrum from gauge bosons decay. On the other hand, given the high dependence of the spectra on the lepton flavour, we allow non-universal lepton contributions. The DM annihilation spectra of all these channels are calculated using micrOMEGAs_3.6 (Bélanger et al. 2011, 2014).

Concerning the case of a leptophilic DM, only three branching ratios are introduced as free parameters. They correspond to the three leptonic channels $\left(\phi \phi \rightarrow 2 \mathrm{e}^{+} 2 \mathrm{e}^{-}, \phi \phi \rightarrow 2 \mu^{+} 2 \mu^{-}, \phi \phi \rightarrow\right.$ $\left.2 \tau^{+} 2 \tau^{-}\right)$, where $\phi$ is a light scalar mediator. In this case the annihilation spectra are taken from the PPPC4DMID (Cirelli et al. 2011; Ciafaloni et al. 2011).

For both cases, the DM component thus depends on the branching ratios, on the DM mass $m_{\chi}$, and on $\langle\sigma v\rangle$ the velocity averaged annihilation cross-section, henceforth loosely dubbed "the cross section".

The search for the best fit to the positron data is led in the following way: for twenty DM masses logarithmically distributed in the range $[100 \mathrm{GeV} ; 1000 \mathrm{GeV}]$, we perform a fit to the AMS02 measurements of the positron flux using MINUIT. We determine the minimum value of the $\chi^{2}$ defined as

$\chi^{2}=\sum_{i}\left\{\frac{\Phi^{\text {data }}\left(E_{i}\right)-\Phi^{\text {th }}\left(E_{i}\right)}{\sigma^{\text {data }}\left(E_{i}\right)}\right\}^{2}$.

In the case of the five annihilation channels, the parameter space is of dimension six: two corresponding to $m_{\chi}$ and $\langle\sigma v\rangle$, and four for the branching ratios $b_{i}$ given the constraint $\sum_{i} b_{i}=1$. In the case of the leptophilic DM, the parameter space is of dimension four. To remain conservative, for each propagation model, we perform the fit seven times, varying the Fisk potential in the $3 \sigma$ range [647 $\mathrm{MV} ; 830 \mathrm{MV}$ ] where $724 \mathrm{MV}$ corresponds to the nominal value of the potential (Ghelfi et al. 2016). In the following, we first discuss results for DM annihilation into the five channels $b \bar{b}, W^{+} W^{-}, \mathrm{e}^{+} \mathrm{e}^{-}, \mu^{+} \mu^{-}$, and $\tau^{+} \tau^{-}$, then for the leptophilic DM case.

\subsection{Results of the analysis}

We plot in Fig. 10 the main result of our analysis, namely the evolution of the $\chi^{2}$ per degrees of freedom $\chi_{\mathrm{d} . \text { f }}^{2}$, as a function of the DM mass $m_{\chi}$. The two plots correspond to DM annihilating into a fitted combination of $b \bar{b}, W^{+} W^{-}, \mathrm{e}^{+} \mathrm{e}^{-}, \mu^{+} \mu^{-}$, and $\tau^{+} \tau^{-}$channels (left panel) and $\phi \phi \rightarrow 2 \mathrm{e}^{+} 2 e^{-}, \phi \phi \rightarrow 2 \mu^{+} 2 \mu^{-}$, and $\phi \phi \rightarrow 2 \tau^{+} 2 \tau^{-}$channels (right panel). The results are displayed for different values of the Fisk potential (nominal value, 
M. Boudaud et al.: The pinching method for Galactic cosmic ray positrons: Implications in the light of precision measurements
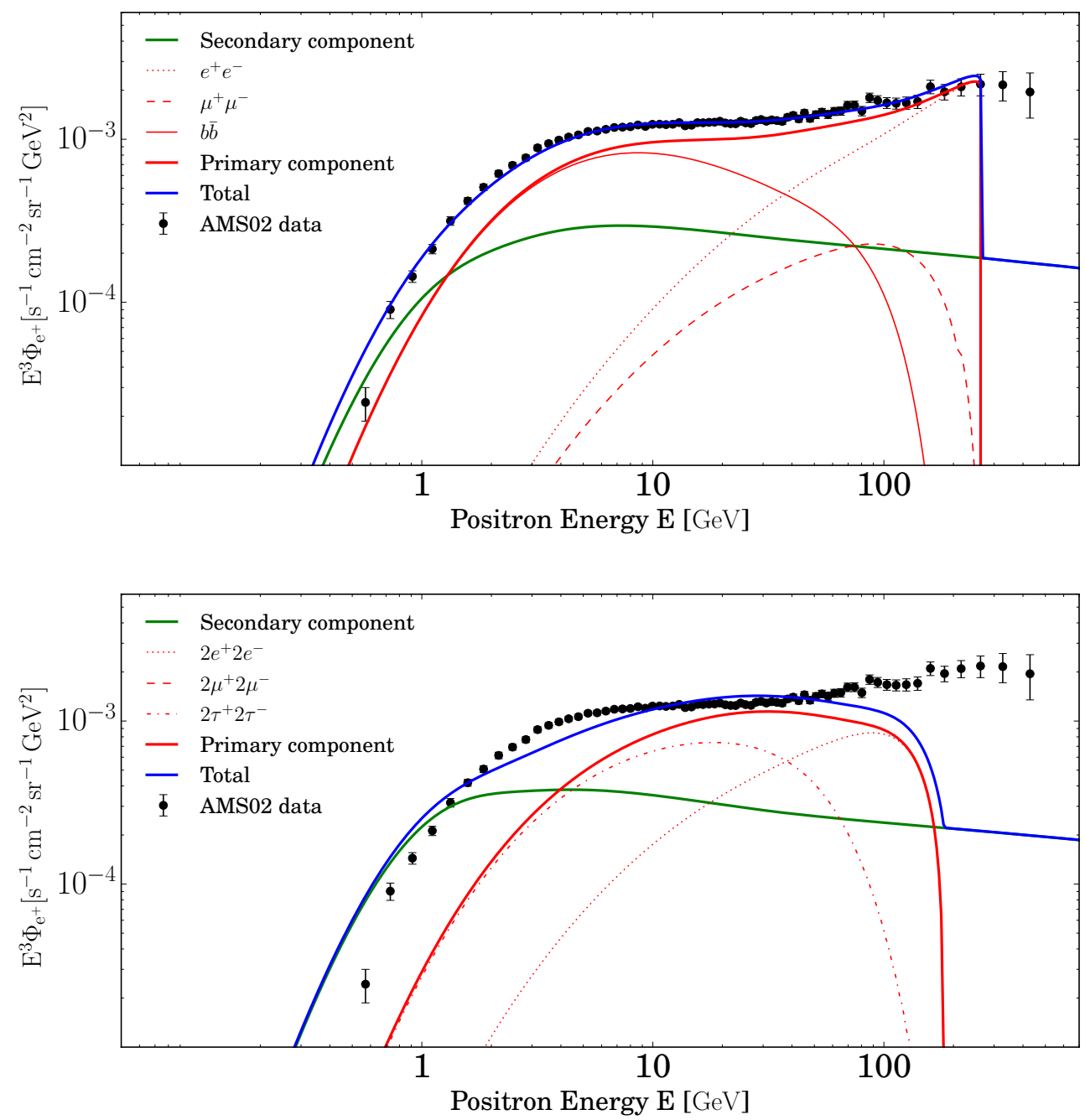

Fig. 11. Global best fit for the direct annihilation into standard model particles (upper panel) and annihilation into four leptons through light mediators (lower panel). The DM (resp. secondary) component is displayed in thick red (resp. thick green) while the total flux, the sum of these two components, is shown in thick blue. In the case of direct annihilation, the electron, muon, and $b$ quark channels are displayed in dotted, dashed, and solid red lines, respectively. In the case of annihilations through light mediators, the electron and $\tau$ channels are displayed in dotted and dot-dashed red lines, respectively. The AMs-02 positron flux, including experimental uncertainties, is superimposed with black dots.

and $\pm 3 \sigma)$. In the direct annihilation case, as one can see from Fig. 10, we find a a global best fit corresponding to a minimal $\chi_{\text {d.o.f. }}^{2}=\chi^{2} / n$ d.o.f. $=100 / 66=1.5$. It is obtained for a DM mass of $m_{\chi}=264 \mathrm{GeV}$ annihilating into $b \bar{b}, \mathrm{e}^{+} \mathrm{e}^{-}$, and $\mu^{+} \mu^{-}$ with branching ratios of $0.92,0.05$, and 0.03 respectively (the branching ratios for the channels $W^{+} W^{-}$and $\tau^{+} \tau^{-}$are found to be zero). The associated annihilation cross-section is $\sim 272$ times larger than the thermal cross-section. This means that a peculiar enhancement mechanism is required, as has been found in many former studies.

Similarly, in the leptophilic case, we find a global best fit associated to a $\chi_{\text {d.o.f. }}^{2}=1231 / 68=18$. This corresponds to a DM mass $m_{\chi}$ of $183 \mathrm{GeV}$ annihilating into $\phi \phi \rightarrow 2 \mathrm{e}^{+} 2 \mathrm{e}^{-}$and $\phi \phi \rightarrow 2 \tau^{+} 2 \tau^{-}$with respective branching ratios of 0.09 and 0.91 . The branching ratio of the channel $\phi \phi \rightarrow 2 \mu^{+} 2 \mu^{-}$is chosen as zero by the fit.

Interestingly, values of the minimal $\chi_{\text {d.o.f. }}^{2}$ are high, especially in the leptophilic case. To understand results of the fitting procedure, we plot in Fig. 11 the theoretical positron fluxes obtained using the best fit models, together with the data. In the direct annihilation case, one can note the remarkably good agrement of the fit with the data up to $300 \mathrm{GeV}$. However, the prediction is in discrepancy with the last two data points at two to four sigma. These two points (and marginally the first one) are responsible for the low quality of the fit yielding a $\chi_{\text {d.o.f. }}^{2}=1.5$ or equivalently a $p$-value of $0.4 \%$. From the left panel of Fig. 10, we observe that imposing the DM mass to be above $450 \mathrm{GeV}$ in order to explain the last two points of the positron flux would yield an even poorer $\chi_{\text {d.o.f. }}^{2}$, above 2 . In the leptophilic case, the picture is even worse: no single part of the spectrum can be accurately described when one tries to fit the whole energy range. Thus, the resulting minimal $\chi_{\text {d.o.f. }}^{2}$ is extremely bad.

Let us now discuss the evolution of $\chi_{\text {d.o.f. }}^{2}$ with respect to the DM mass. First of all, we observe that, whatever the solar modulation, the evolution of the $\chi_{\text {d.o.f. }}^{2}$ is similar: with increasing DM mass, the $\chi_{\text {d.o.f. }}^{2}$ first decreases, reaching a minimal values around a few hundred $\mathrm{GeV}$, and then increases. Low DM masses cannot account for the high-energy part of the positron flux since no positrons with energy above the DM mass can be emitted. Thus, at first, the goodness of the fit is improving (i.e.

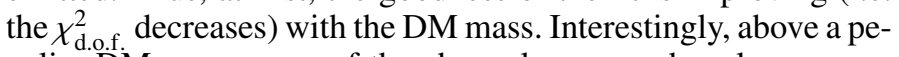
culiar DM mass, none of the channels can produce low-energy positrons in a sufficient amount to explain the low-energy part of the data. Consequently the goodness of fit degrades, that is, 
Table 5. Number of propagation models allowed after the analysis of Sect. 4, and associated parameter ranges.

\begin{tabular}{ccccccc}
\hline \hline & $\begin{array}{c}\text { Allowed } \\
\text { propagation models }\end{array}$ & $\delta$ & $\begin{array}{c}K_{0} \\
\left(\mathrm{kpc}^{2} \mathrm{Myr}^{-1}\right)\end{array}$ & $\begin{array}{c}L \\
(\mathrm{kpc})\end{array}$ & $\begin{array}{c}V_{\mathrm{c}} \\
\left(\mathrm{km} \mathrm{s}^{-1}\right)\end{array}$ & $\begin{array}{c}V_{\mathrm{a}} \\
\left(\mathrm{km} \mathrm{s}^{-1}\right)\end{array}$ \\
\hline $\begin{array}{c}\text { All data points } \\
\text { First point excluded }\end{array}$ & 54 & 0.46 & $0.0599-0.0764$ & $8.5-15$ & $5-6$ & $104.0-118.3$ \\
First two points excluded & 623 & $0.46-0.7$ & $0.0240-0.0764$ & $4.5-15$ & $5-12$ & $70.9-119.0$ \\
First three points excluded & 623 & $0.46-0.7$ & $0.0240-0.0764$ & $4.5-15$ & $5-12$ & $70.9-119.0$ \\
\hline
\end{tabular}

Notes. We present results of the skimming method discarding successively up to the three first data points.

the $\chi_{\text {d.o.f. }}^{2}$ increases. As a result, there is a "middle ground" at a peculiar mass (the value changes with annihilation channels and Fisk potential), which corresponds to the best possible attempt to fulfill similarly high- and low-energy constraints. Somehow, the flatness of the spectrum is such that it is not possible to accommodate it entirely with a single primary component. We also note the drift of the best fit towards lower DM masses as the solar modulation increases. This is simply because the lowenergy part of the fluxes is more and more suppressed with an increasing Fisk potential. Hence, additional low-energy positrons are needed (i.e. lighter DM) to fit the data. However increasing the Fisk potential is not necessary associated with an improving $\chi_{\text {d.o.f. }}^{2}$. The actual shape of the annihilation spectrum matters, as can be seen by comparing the upper and lower panels of Fig. 11. Indeed, in the direct annihilation case, increasing the Fisk potential tends to improve the fit, whereas in the case of annihilation through light mediators it worsens it.

In summary, we find interpretation of the excess in terms of pure DM annihilations challenging, since our conservative analysis always leads to low-quality fits of the data. It is remarkable that the shape of the positron excess, with respect to the pure secondary prediction, cannot be captured by annihilations of a single DM species. This feature is due to: i) the high precision of the AMS-02 data, and ii) the possibility to fit the whole data range thanks to our new semi-analytical resolution method. It is reasonable to believe that a fit above $10 \mathrm{GeV}$ would not have had this issue. Similarly, we expect multi-component models, with, for example, one heavy and one light DM species to be able to fit the excess. In the following section, we discuss how robust this conclusion is against a relaxation of our selection criterion of propagation parameters, as well as the inclusion of theoretical uncertainties in the modelling.

\section{Robustness of the results}

In this section, we assess the robustness of the conclusions drawn above, under changes in the skimming method and source-term modelisation. Since our selection criterion does not combine information of data points together but makes use of them separetely, we investigate first whether or not a specific data point of the positron flux could be leading the constraints, eventually biasing the results. Indeed, one can see in Fig. 8 that the position of the first data point measured by AMS-02 is very low with respect to the expected trend from the predicted secondary positron flux. Secondly, we evaluate uncertainties of the secondary component source term in order to attest that they can be safely neglected in our analysis. These uncertainties come from the experimental measurement of the AMS-02 primary fluxes, as well as the choice of $p$ and He spallation cross-section onto the ISM.

To check whether the first data point is more discriminating than the higher energy ones, we repeat the skimming method presented in Sect. 4 discarding this peculiar point from the analy- sis. The comparison between the results of the analysis with and without the first point is reported in the first two lines of Table 5. Not surprisingly, the number of selected models does increase. However, in a much more striking way than expected, we notice that it increases more than twelve times. The parameter space counts now 623 allowed models. We conclude that within our skimming method, the first point of the flux has indeed a very strong discriminating power. To check that it was a pecularity of the first data point, we successively repeat the skimming method discarding up to the three first data points. The results are reported in Table 5 and confirm the singularity of the first point; the number of allowed models never exceeds 692. Let us emphasise that even without its first point, the positron flux provides stringent constraints on propagation parameters; it enables to rule out two thirds of the parameter space allowed by former boron-over-carbon analysis. To check the impact of a bigger parameter space on our DM analysis (see Sect. 5), we repeat it with the 623 propagation models selected without the first point of the positron flux. In the case of DM annihilating directly into a combination of $b \bar{b}, W^{+} W^{-}, \mathrm{e}^{+} \mathrm{e}^{-}, \mu^{+} \mu^{-}$, and $\tau^{+} \tau^{-}$channels, the $\chi_{\text {d.o.f. }}^{2}$ of the best fit is now of 1.1 , which corresponds to a $p$-value of $26 \%$. Such a value might indicate that DM annihilation can still explain the positron excess. However, the associated $\mathrm{DM}$ mass is $336 \mathrm{GeV}$, causing a cut-off of the primary positron flux at this energy, not observed in AMs-02 data. Hence, with improving statistics in these last two bins, it is likely that the $\chi_{\text {d.o.f. }}^{2}$ will quickly degrade. On the other hand, imposing the DM mass to be above the energy of the last data point increases the $\chi_{\text {d.o.f. }}^{2}$ to a value above 2 , indicative of a bad quality fit. In the hypothesis of leptophilic DM annihilating into $\phi \phi \rightarrow 2 \mathrm{e}^{+} 2 \mathrm{e}^{-}$ and $\phi \phi \rightarrow 2 \tau^{+} 2 \tau^{-}$through a light mediator, the best fit has a $\chi_{\text {d.o.f. }}^{2}$ greater than 10 . Thus, the conclusion remains unaltered. We now turn to assessing the impact of uncertainties associated to the source term of the secondary component on our conclusions. A key ingredient of the secondary positron prediction is an accurate measurement of the flux of their progenitors; mainly proton and helium nuclei. In Sect. 3.1, we gave the parameterisation used to describe these fluxes, as well as the best-fit value of the parameters. Given the finite precision of AMS-02 measurements, uncertainties in the determination of these parameters can affect our secondary positron prediction. To estimate the uncertainty associated to the fitting procedure, we developed an original method that takes into account both systematic and statistical uncertainties of the measured primary fluxes. We proceed in the following way: we first generate mock data of the primary fluxes within their total uncertainties, fit them with our parameterisation, and compute a new secondary positron flux. Repeating this process 10000 times allows us to determine the distribution of the secondary positron flux in each energy bin. The mock data for the primary fluxes are generated according to the following strategy: for each data point a new random value is computed as $\bar{\Phi}^{\text {data }}\left(E_{i}\right)+\delta \Phi^{\text {stat }}\left(E_{i}\right)+\delta \Phi^{\text {syst }}\left(E_{i}\right)$, where $\bar{\Phi}^{\text {data }}$ is the mean 
M. Boudaud et al.: The pinching method for Galactic cosmic ray positrons: Implications in the light of precision measurements
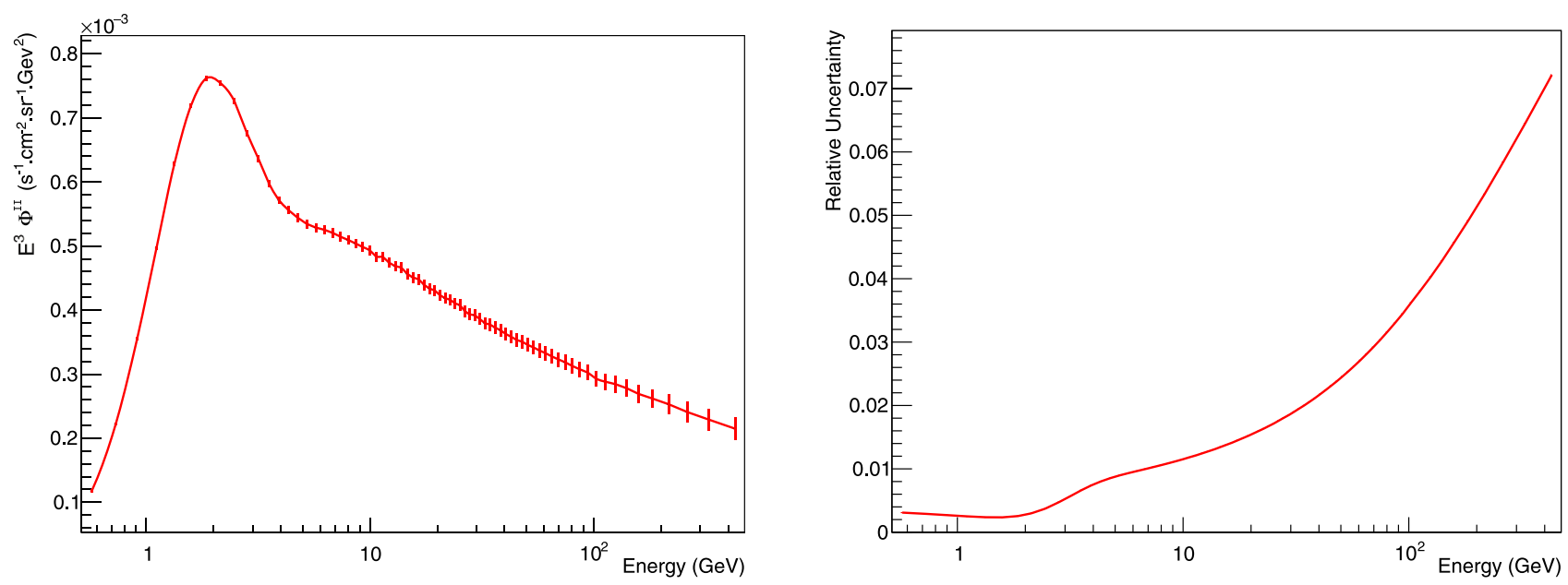

Fig. 12. Left panel: secondary positron flux (multiplied by $E^{3}$ ) as a function of the positron energy. The error bars represent the uncertainty due to the experimental uncertainty on the proton and helium fluxes. Right panel: relative uncertainty on the secondary positron flux, as a function of the positron energy.

value of the flux in the energy bin $E_{i}, \delta \Phi^{\text {stat }}$ is drawn from a Gaussian distribution with standard deviation $\sigma^{\text {stat }}\left(E_{i}\right)$ and $\delta \Phi^{\text {syst }}$ is drawn from a uniform distribution of size $2 \sigma^{\text {syst }}\left(E_{i}\right)$. These two uncertainties $\sigma$ are provided by the AMs- 02 collaboration in Aguilar et al. (2015b,a). Results are displayed in Fig. 12. On the left panel is shown the distribution of our prediction in each energy bin, compared to the fiducial value calculated with the MED propagation model. The relative uncertainty displayed in the right panel is found to increase with the energy, with a maximum of $7 \%$ at $500 \mathrm{GeV}$. The experimental uncertainties of the positron flux are respectively of $6 \%$ and $30 \%$, much larger than the theoretical uncertainty yielded by the primary fluxes. We thus conclude that the precision in the measurement of the primary fluxes is sufficiently small that it does not alter our analysis.

A second major ingredient entering the source term for secondary positrons are the cross-sections adopted for the $p$ and He interaction with the ISM. In our studies, we used protonproton cross-section from Kamae et al. (2006). We recall that any other nucleus-nucleus cross-section can be obtained by rescaling this one with an empirical factor, which we took from Norbury \& Townsend (2007). The choice of proton-proton cross-section from Kamae et al. (2006) is motivated by the fact that, at low energy, this model produces less positrons than the commonly used Moskalenko \& Strong (1998), which includes the parameterisation of the Lorentz invariant obtained by Tan \& Ng (1983) and Badhwar et al. (1977). We therefore adopt a strategy similar to our treatment of solar modulation, which minimises as much as possible the positron flux below $10 \mathrm{GeV}$ by using a very high Fisk potential, on the edge of currently allowed values. Although there is an uncertainty associated to the cross-section and solar modulation modelling, our choices lead to conservative results and thus robust conclusions.

\section{Conclusion}

Two years ago, the AMS-02 collaboration released the most precise measurement of the positron flux in the energy range 0.5 to $500 \mathrm{GeV}$, confirming the high-energy excess with respect to pure secondary predictions. Until now, most of the studies trying to explain this excess in terms of DM annihilations restricted themselves to energies above $10 \mathrm{GeV}$ by prejudice and to simplify computations. Indeed, below this energy, several mechanisms taking place in the halo, namely diffusive reacceleration and convection, as well as energy losses in the disc, make the resolution of the propagation equation much more involved. However, a consistent model should be able to explain the positron flux over the entire energy range covered by the AMS-02 data.

We have therefore reinvestigated the problem of the positron anomaly with a new semi-analytical resolution method enabling us to take into account transport processes so far neglected but important below a few GeV. The key idea is to pinch energy losses occurring in the whole magnetic halo, namely inverse Compton and synchrotron, inside the Galactic disc. The corresponding energy loss rate is artificially enhanced by a so-called pinching factor, which is calculated for each energy. This allows us to solve the CR propagation equation using a Bessel expansion and the Cranck-Nicholson scheme.

With this procedure, we recover the correct high-energy positron flux at the per mille level and extend the computation to low energies, at the main advantage of a very fast resolution technique compared to fully numerical methods. We have re-evaluated both primary and secondary components of the positron flux, finding as expected that they are significantly affected at low energies by the incorporation of the thus far neglected CR transport mechanisms. Surprisingly, we also find that modifications are still substantial at a few tens of $\mathrm{GeV}$, depending on the CR propagation parameters. As pointed out in Delahaye et al. (2009) and Lavalle et al. (2014), we confirm that the secondary prediction of the positron flux at low energies can be in large excess compared to AMS-02 data, even for propagation models compatible with the $\mathrm{B} / \mathrm{C}$ analysis from Maurin et al. (2001).

Motivated by this result, we have carried out a scan over the CR propagation parameters of Maurin et al. (2001), applying an original skimming method which leads to severe constraints on the propagation parameters: out of the 1623 models, only 54 survive the procedure. In particular, the benchmark MIN and MED configurations are excluded. On the other hand, MAXlike propagation models, that is, with large $\left\{K_{0}, L\right\}$ and small $\delta$, are favoured by the data. Those models are very close to the best configuration found in Kappl et al. (2015) by fitting the preliminary $\mathrm{B} / \mathrm{C}$ data of AMS-02. This needs to be confirmed with the newest B/C published recently by AMs-02 (Aguilar et al. 2016b). We do not expect major changes in our conclusions.

To overcome the difficulty arising from solar modulation, we have made use of a very high Fisk potential, $3 \sigma$ above the mean 
value obtained by Ghelfi et al. (2016). This choice minimises the flux at low energies and makes our skimming procedure of the CR parameter space conservative, leading us to keep models that should be disregarded.

In a similar way, we have made use of the $p$ and He spallation cross-sections from Kamae et al. (2006) since they lead to the lowest amount of positrons. Furthermore, we have checked that uncertainties in the measurements of the $p$ and He fluxes do not alter our result. Finally, given that our skimming method makes use of information from data points separately, we have investigated whether or not a specific data point could drive the constraints. We found that this is indeed the case. The first data point has a much higher discriminating power than the others. Discarding it from the analysis, our skimming method selected 623 models, which still corresponds to a rejection of about two thirds of the parameter space. We can therefore conclude that the positron flux is a very useful and independent probe of CR propagation, to be used in synergy with other tracers such as the $\mathrm{B} / \mathrm{C}$ ratio. Our results also indicate that the positron excess is already present at $\mathrm{GeV}$ energies, typically starting above $2 \mathrm{GeV}$.

Finally, we have re-investigated the explanation of the positron anomaly in terms of annihilations of a single DM species, in the WIMP framework, over the whole energy range of the AMS-02 data. We have performed a scan over WIMP mass and explored the possibility of: i) direct annihilation into a combination of channels; and ii) leptophilic DM annihilating into four leptons through a light mediator. For a given WIMP mass and propagation model (selected by our skimming method), we have obtained the best-fit values of the annihilation cross-section and branching ratios. Our most striking result is that no good fit to the data is obtained for either case i) or ii). Indeed, in case i), the best fit is found for a mass of $264 \mathrm{GeV}$ that does not allow reproduction of the highest-energy data points. Hence, the associated $p$-value is as low as $0.4 \%$. On the other hand, requiring the DM mass to be larger than $500 \mathrm{GeV}$ yields $\chi_{\text {d.o.f. }}^{2}>3$, since the low-energy part of the data cannot be consistently accommodated. Case ii) turns out to be even worse, the best-fit $\chi_{\text {d.o.f }}^{2}$ being as high as 15 . We have checked the robustness of our conclusions against a few possible loopholes.

We are thus led to the conclusion that annihilations of a single DM species should be disregarded as the sole origin of the positron excess, on the basis of the positron data themselves, irrespective of other observables such as the antiproton flux or CMB anisotropies. It is likely that more ad-hoc multi-species models, with, for example, one heavy and one light DM particle, will be able to accommodate the excess, although a strong statement would require a dedicated study. It is probable that such an analysis, with a unique pulsar as the source of the anomaly, would lead to similar conclusions, requiring in the future more realistic multi-component studies.

Acknowledgements. We would like to thank Pasquale D. Serpico, Richard Taillet and Eric Ragoucy for enlightening discussions in the initial stages of this work. Part of this work was supported by the French Institut universitaire de France, by the French Agence Nationale de la Recherche under contract 12-BS05-0006 DMAstroLHC, by the Investissements d'avenir, Labex ENIGMASS, by the CNES, France, and by the PRC (Projet de Recherche Conjoint) CNRS-FAPESP. M.V. and E.F.B. are grateful to the São Paulo Research Foundation (FAPESP) for the support received through grants Nos. 2014/19149-7, 2014/50747-8 and 2015/13533-2.

\section{References}

Accardo, L., Aguilar, M., Aisa, D., et al. 2014, Phys. Rev. Lett., 113, 121101 Ackermann, M., Ajello, M., Albert, A., et al. 2012, Phys. Rev. D, 85, 109901
Adriani, O., Barbarino, G. C., Bazilevskaya, G. A., et al. 2009, Nature, 458, 607 Aguilar, M., Alberti, G., Alpat, B., et al. 2013, Phys. Rev. Lett., 110, 141102 Aguilar, M., Aisa, D., Alvino, A., et al. 2014, Phys. Rev. Lett., 113, 121102 Aguilar, M., Aisa, D., Alpat, B., et al. 2015a, Phys. Rev. Lett., 115, 211101 Aguilar, M., Aisa, D., Alpat, B., et al. 2015b, Phys. Rev. Lett., 114, 171103 Aguilar, M., Ali Cavasonza, L., Alpat, B., et al. 2016a, Phys. Rev. Lett., 117, 091103

Aguilar, M., Ali Cavasonza, L., Ambrosi, G., et al. 2016b, Phys. Rev. Lett., 117, 231102

Aguilar, M., Ali Cavasonza, L., Ambrosi, G., et al. 2016c, Phys. Rev. Lett., 117, 231102

Badhwar, G. D., Golden, R. L., \& Stephens, S. A. 1977, Phys. Rev. D, 15, 820 Baltz, E. A., \& Edsjö, J. 1999, Phys. Rev. D, 59, 023511

Barwick, S. W., Beatty, J. J., Bhattacharyya, A., et al. 1997, ApJ, 482, L191 Beatty, J. J., Bhattacharyya, A., Bower, C., et al. 2004, Phys. Rev. Lett., 93, 241102

Bélanger, G., Boudjema, F., Brun, P., et al. 2011, Comput. Phys. Commun., 182, 842

Bélanger, G., Boudjema, F., Pukhov, A., \& Semenov, A. 2014, Comput. Phys. Commun., 185, 960

Blasi, P. 2009, Phys. Rev. Lett., 103, 051104

Boudaud, M., Aupetit, S., Caroff, S., et al. 2015, A\&A, 575, A67

Bovy, J., \& Tremaine, S. 2012, ApJ, 756, 89

Casse, F., Lemoine, M., \& Pelletier, G. 2002, Phys. Rev. D, 65, 023002

Ciafaloni, P., Comelli, D., Riotto, A., et al. 2011, J. Cosmol. Astropart. Phys., 3, 019

Cirelli, M., Corcella, G., Hektor, A., et al. 2011, J. Cosmol. Astropart. Phys., 3, 051

Coste, B., Derome, L., Maurin, D., \& Putze, A. 2012, A\&A, 539, A88

Delahaye, T., Lineros, R., Donato, F., Fornengo, N., \& Salati, P. 2008 Phys. Rev. D, 77, 063527

Delahaye, T., Lineros, R., Donato, F., et al. 2009, A\&A, 501, 821

Delahaye, T., Lavalle, J., Lineros, R., Donato, F., \& Fornengo, N. 2010, A\&A 524, A51

Di Bernardo, G., Evoli, C., Gaggero, D., Grasso, D., \& Maccione, L. 2013, J. Cosmol. Astropart. Phys., 3, 36

Di Mauro, M., Donato, F., Fornengo, N., Lineros, R., \& Vittino, A. 2014, J. Cosmol. Astropart. Phys., 4, 6

Di Mauro, M., Donato, F., Fornengo, N., \& Vittino, A. 2016, J. Cosmol. Astropart. Phys., 5, 031

Donato, F., Fornengo, N., Maurin, D., Salati, P., \& Taillet, R. 2004, Phys. Rev. D, 69, 063501

DuVernois, M. A., Barwick, S. W., Beatty, J. J., et al. 2001, ApJ, 559, 296

Ferrière, K. M. 2001, Rev. Mod. Phys., 73, 1031

Fisk, L. A. 1971, J. Geophys. Res., 76, 221

Genolini, Y, Putze, A., Salati, P., \& Serpico, P. D. 2015, A\&A, 580, A9

Genolini, Y., Salati, P., Serpico, P., \& Taillet, R. 2017, A\&A, 600, A68

Ghelfi, A., Barao, F., Derome, L., \& Maurin, D. 2016, A\&A, 591, A94

Giesen, G., Boudaud, M., Génolini, Y., et al. 2015, J. Cosmol. Astropart. Phys., 9, 023

Hooper, D., Blasi, P., \& Dario Serpico, P. 2009, J. Cosmol. Astropart. Phys., 1, 25

Kamae, T., Karlsson, N., Mizuno, T., Abe, T., \& Koi, T. 2006, ApJ, 647, 692

Kappl, R., Reinert, A., \& Winkler, M. W. 2015, J. Cosmol. Astropart. Phys., 10, 034

Korsmeier, M., \& Cuoco, A. 2016, Phys. Rev. D, 94, 123019

Lavalle, J., Maurin, D., \& Putze, A. 2014, Phys. Rev. D, 90, 081301

Lin, S.-J., Yuan, Q., \& Bi, X.-J. 2015, Phys. Rev. D, 91, 063508

Linden, T., \& Profumo, S. 2013, ApJ, 772, 18

Maurin, D., Donato, F., Taillet, R., \& Salati, P. 2001, ApJ, 555, 585

Mertsch, P., \& Sarkar, S. 2014, Phys. Rev. D, 90, 061301

Moskalenko, I. V., \& Strong, A. W. 1998, ApJ, 493, 694

Navarro, J. F., Frenk, C. S., \& White, S. D. M. 1997, ApJ, 490, 493

Norbury, J. W., \& Townsend, L. W. 2007, Nucl. Instr. Meth. Phys. Res. B, 254 187

Profumo, S. 2012, Central Eur. J. Phys., 10,

Ptuskin, V. S., Voelk, H. J., Zirakashvili, V. N., \& Breitschwerdt, D. 1997, A\&A, 321, 434

Putze, A., Derome, L., \& Maurin, D. 2010, A\&A, 516, A66

Strong, A. W., \& Moskalenko, I. V. 1998, ApJ, 509, 212

Strong, A. W., \& Moskalenko, I. V. 2001, Adv. Space Res., 27, 717

Tan, L. C., \& Ng, L. K. 1983, J. Phys. G Nucl. Phys., 9, 1289

Yoon, Y. S., Ahn, H. S., Allison, P. S., et al. 2011, ApJ, 728, 122 